Commun. Math. Phys. 133, 91-117 (1990)

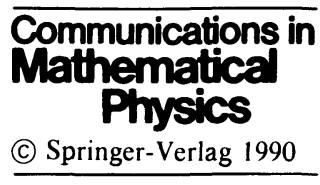

\title{
Relating the Approaches to Quantised Algebras and Quantum Groups
}

\author{
Nigel Burroughs * \\ Department of Applied Mathematics and Theoretical Physics, Silver Street, Cambridge, England \\ CB3 9EW
}

Received July 26, 1989; in revised form January 24, 1990

\begin{abstract}
This paper constructs two representations of the quantum group $U_{q} g^{\prime}$ by exploiting its quotient structure and the quantum double construction. Here the quantum group is taken as the dual to the quantised algebra $U_{q} g$, a one parameter deformation of the universal enveloping algebra of the Lie algebra $g$, as in Drinfel'd [6] and Jimbo [10]. From the two representations, the Hopf structure of the quantised algebra $U_{q} g$ is reexpressed in a matrix format. This is the very structure given by Faddeev et al. [7], in their approach to defining quantum groups and quantised algebras via the quantisation of the function space of the associated Lie group to $g$.
\end{abstract}

\section{Introduction}

A newcomer to the field of quantum groups will encounter four essential papers on the structure and definition of quantised algebras and quantum groups, namely those by Jimbo [9, 10], Drinfel'd [6] and Faddeev et al. [7]. These works define the concepts of quantised algebras and quantum groups using two alternative approaches. The first two authors use a more mathematical formulation for defining a quantised algebra, introducing a one parameter deformation of an universal enveloping algebra of a Lie (or Kac Moody) algebra. The concepts are rather intricate, and for this reason the approach of Faddeev et al. [7] - based on a quantisation of the function space of the accompanying Lie group-may well be more appealing initially, especially to the physics community. However the two approaches remain rather disjoint, the connection between the two being elusive, the reader only having claims of their equivalence in [7].

As discussed in Drinfel'd [6], the motivation for introducing the one parameter deformation of the universal enveloping algebra comes from the classical isomorphism between the function space of the (connected) Lie group $G$ and the

\footnotetext{
^ Supported by a SERC studentship
} 
universal enveloping algebra of the associated Lie algebra $g^{1}$ :

$$
U g^{\prime} \cong \operatorname{Fun}(G)
$$

i.e. any function on the group $G$ defines a linear mapping of the universal enveloping algebra $U g$ and vice versa. This isomorphism is between Hopf algebras. The subtleties of this isomorphism will be ignored in this paper, see [6]. Thus a deformation/quantisation ${ }^{2}$ of the function space should also produce a deformation of the universal enveloping algebra. Hence on the quantum level we obtain the isomorphism $[6,7]$ :

$$
U_{q} g^{\prime} \cong \operatorname{Fun}_{q}(G)=\operatorname{Fun}\left(G_{q}\right)
$$

where $U_{q} g$ denotes the quantised algebra, [6], $\operatorname{Fun}_{q}(G)$ the quantised functions on the Lie group $G$, [7], $G_{q}$ the quantum formal group corresponding to $G$, [17] and $\operatorname{Fun}\left(G_{q}\right)$ the functions on $G_{q}[17]$. The quantised algebra, $U_{q} g$ and quantised function space, $\operatorname{Fun}_{q}(G)$ are defined as follows:

Quantised Algebra. We shall follow Drinfel'd [6] in defining the quantisation of a Lie algebra. For a general Lie algebra $g$, with a system of simple roots $S$, the quantised algebra $U_{q} g$ is an Hopf algebra over the ring $C[[h]]$, that is, a one parameter deformation of the universal enveloping algebra $U g$. It is generated by $\left\{1, H_{i}, X_{i}^{ \pm}\right\}$; three generators for each simple root $\alpha_{i}$ in $S$, the $H_{i}$ corresponding to the coroots of $g$. These generators have the following Hopf structure:

$$
\begin{aligned}
{\left[H_{i}, X_{j}^{ \pm}\right] } & = \pm a_{i j} X_{j}^{ \pm}, \quad\left[X_{i}^{+}, X_{j}^{-}\right]=\delta_{i j} \frac{\sinh \left(\frac{h}{2} H_{i}\right)}{\sinh \left(\frac{h}{2}\right)}, \\
\Delta\left(H_{i}\right) & =1 \otimes H_{i}+H_{i} \otimes 1, \quad \Delta\left(X_{i}^{ \pm}\right)=X_{i}^{ \pm} \otimes q^{H_{i} / 2}+q^{-H_{i} / 2} \otimes X_{i}^{ \pm} .
\end{aligned}
$$

Here $a_{i j}$ is the Cartan matrix of $g$. The generators $X_{i}^{ \pm}$are also required to satisfy the $q$-analogue Serre relations given in [6] and [10]. However by introducing generators for each root of $g$, the use of these Serre relations can be avoided $[2,14]$. These quantisations are in fact quasi-triangular Hopf algebras [6], that is, there exists an universal $R$-matrix which will be denoted by $R$. This is an element of $U_{q} g \otimes U_{q} g$. The quantised algebra $U_{q} g$ as defined by Jimbo, [10] differs from the above construction in that it is an Hopf subalgebra of the above, only the combination $k_{i}=q^{H_{i} / 2}$ occurring and not $H_{i}$ itself. This Hopf algebra is only pseudo quasi-triangular [4, 6]. The approach of Faddeev et al. [7] reproduces the quantised algebra as defined by Jimbo [10].

The quantum group corresponding to the quantised algebra $U_{q} g$ is defined as

\footnotetext{
1 A prime denotes the linear dual (Hopf). Fun is used to denote the $C^{\infty}$ functions.

2 The deformation parameter is called Planck's constant in analogy with the quantisation of classical mechanics, and denoted $h$. The quantity $q=e^{h / 2}$ is found to be useful
} 
the Hopf dual ${ }^{3}$ to $U_{q} g$, this definition being natural in the framework of pseudo-groups as defined by Woronowicz [16]. From relation (1), the quantum

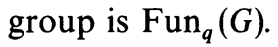

Quantised Function Spaces. Contrast the above definition of quantised algebras and quantum groups with that of Faddeev et al. [7] and Woronowicz [16]. This construction assumes the existence of a matrix $R$ (not to be confused with the universal $R$-matrix), that is valued in $\operatorname{End}(V \otimes V)$, where $V$ is some $n$ dimensional vector space over the ring $C[[h]] . R$ is assumed to satisfy the Quantum Yang Baxter equation (QYBE):

$$
R_{12} R_{13} R_{23}=R_{23} R_{13} R_{12}
$$

An Hopf algebra $A(R)$ is then defined with generators $\left\{1, t_{i j}\right\}$ satisfying the following relations:

$$
\begin{aligned}
R T_{1} T_{2} & =T_{2} T_{1} R \\
\Delta\left(t_{i j}\right) & =\sum_{k} t_{i k} \otimes t_{k j}
\end{aligned}
$$

Here the matrix $T$ is a matrix of generators: $(T)_{i j}=t_{i j}$. For this algebra, the QYBE (3) corresponds to an associativity condition. For $R$ in the fundamental representation of $U_{q} s l(n)$, the Hopf algebra $A(R)$ can be considered as the quantised function space of $G l(n)$, i.e. $\operatorname{Fun}_{q}(G l(n))$, [7]. An application of a quantum determinant condition reduces this to $\operatorname{Fun}_{q}(\operatorname{Sl}(n))$.

From the algebra $A(R)$, an Hopf algebra $U(R)$ is defined, a subalgebra of the dual to $A(R) . U(R)$ is generated by $\left\{1, l_{i j}^{( \pm)}\right\}$, which are defined by the following evaluations:

$$
\left(L^{( \pm)}, T_{1} \ldots T_{k}\right)=R_{1}^{( \pm)} \ldots R_{k}^{( \pm)}
$$

where the matrix of generators $L^{( \pm)}$is defined as $\left(L^{( \pm)}\right)_{i j}=l_{i j}^{( \pm)}$. The two matrices $R^{( \pm)}$are: $R^{(+)}=P R P, R^{(-)}=R^{-1}$, with $P$ being the transposition matrix on the two factors $V \otimes V$. In fact these generators do not freely generate $U(R)$, see [7] and Sect. 4. By manipulating duality and the evaluation structure in (5), it can be shown that the Hopf structure:

$$
\begin{aligned}
R_{21} L_{1}^{( \pm)} L_{2}^{( \pm)} & =L_{2}^{( \pm)} L_{1}^{( \pm)} R_{21}, \quad R_{21} L_{1}^{(+)} L_{2}^{(-)}=L_{2}^{(-)} L_{1}^{(+)} R_{21}, \\
\Delta\left(l_{i j}^{( \pm)}\right) & =\sum_{k} l_{i k}^{( \pm)} \otimes l_{k j}^{( \pm)}
\end{aligned}
$$

is obtained for the generators $l_{i j}^{( \pm)}[7]$.

From the isomorphism (1), the Hopf algebra $U(R)$ should be the quantised algebra $U_{q} s l(n)$, if $R$ is the $R$-matrix for $U_{q} s l(n)$ in the fundamental representation. In this paper this is proved by obtaining an explicit isomorphism between the

3 Due to the problems of dualising a tensor product, the dual to $U_{q} g$ is not necessarily an Hopf algebra. We define the dual Hopf algebra in terms of the Hopf structure induced on a dual basis of the associated ring module 
generators $\left\{l_{i j}^{ \pm}\right\}$and $\left\{q^{ \pm H_{k} / 2}, X_{l}^{ \pm}\right\}$, i.e. $U(R)$ is the quantised algebra $U_{q} s l(n)$ as defined by Jimbo [10].

The essential observation of our construction is the fact that the Hopf structure of the $T$ generators (4) follows from a representation of the quantised algebra $U_{q} g$, Sect. 1. On observing that the Hopf structure of the algebra $U(R),(6)$ is very similar to that in (4), it may be expected that this will follow from a representation of the dual to $U_{q} g$. In fact two representations are required, one for each of the matrices $L^{( \pm)}$. To reproduce the structure in (6), specific representations need to be chosen. These are chosen in Sect. 3 by observing that the matrices $L^{( \pm)}$can be expressed in terms of the universal $R$-matrix of $U_{q} g(15),(16)$ :

$$
L_{i j}^{(+)}=\operatorname{Id} \otimes \rho_{i j}\left(R_{U_{q} g}\right) \in U_{q} b_{+}, \quad L_{i j}^{(-)}=\rho_{i j} \otimes \operatorname{Id}\left(R_{U_{q} g}^{-1}\right) \in U_{q} b_{-} .
$$

The Hopf structure satisfied by these generators, (6) follows from the representations used, this being derived in Sects. 3 and 7; the mixed relation in $L^{( \pm)}$being derived from the dual of the quantum double in Sect. 7. On restricting to the Lie algebra $s l(n)$, we prove that $\left\{t_{i j}\right\}$ and $\left\{l_{i j}^{ \pm}\right\}$do not generate Fun $_{q}(S l(n))$ and $U_{q} s l(n)$ freely, quantum determinant relations holding on the matrices $T, L^{( \pm)}$, and a diagonal relation between $L^{( \pm)}$. Section 6 illustrates the construction with $U_{q} s l(2)$. The dual to the quantum double is introduced in Sect. 7, this producing the possibility of constructing the quantum double from the quantum group $U_{q} g^{\prime}$. Section 8 encodes the Hopf structure of the quantised algebras $U_{q} s l(n)$ into a matrix format by employing the construction of Sect. 3 and the universal $R$-matrices of $U_{q} s l(n)$, as derived in $[2,14]$. This also allows a systematic construction of all the commutation relations of the generators of $U_{q} s l(n)$ as used in [2].

In the following sections, the notation $l_{i j}^{( \pm)}$will not be employed for the generators of $U_{q} g$ in the matrix formulation. The symbol $\sigma_{i j}^{ \pm}$is preferred.

\section{Algebra Representation Structures of Hope Algebras}

In this section an Hopf subalgebra of the dual to an Hopf algebra $A$ will be defined via an algebra representation of $A$. The commutation relations are expressed in a matrix form by defining a matrix valued in the dual to $A$. The resulting Hopf algebra is identical to that used to define a quantum group in [7].

Consider a quasi-triangular Hopf algebra $(A, R)[6]$ consisting of an Hopf algebra $A$ over the ring $K=C[[h]]$, and an universal $R$-matrix $R \in A \otimes A$ that relates the two coalgebra maps $\Delta$ and $T \circ \Delta$ :

$$
T \circ \Delta(a) R=R \Delta(a), \quad \forall a \in A .
$$

Let there be an algebra representation of $A$ in an $n$ dimensional $K$-module $V$, $\rho: A \rightarrow \operatorname{End}(V) \cong \operatorname{Mat}(n, K)$. The individual matrix elements $\rho_{i j}$ define a mapping $\rho_{i j}: A \rightarrow K$ via the evaluation $a \rightarrow(\rho(a))_{i j}$, and hence $\rho_{i j} \in A^{\prime}$, the dual of $A$. The $\rho_{i j}$ will generate an Hopf subalgebra of $A^{\prime}$ which will be denoted by $A(R)$, following [7]. The Hopf structure of $A(R)$ is induced from the Hopf structure of $A$ by duality, the coalgebra being given by:

$$
\left(\Delta \rho_{i j}, a \otimes b\right) \underset{\operatorname{def}}{=}\left(\rho_{i j}, a b\right)=\sum_{k} \rho_{i k}(a) \rho_{k j}(b) .
$$


The fact that $\rho$ is an algebra representation is necessary here, in order to expand the product of two elements of $A$. Since $a, b$ are arbitrary, we deduce that:

$$
\Delta \rho_{i j}=\sum_{k} \rho_{i k} \otimes \rho_{k j} .
$$

To derive the algebra structure, we shall exploit the fact that $A$ is quasi-triangular. From (7) the universal $R$-matrix relates the two coalgebras of $A$, the very structures that induce the commutation relations of the dual:

$$
\rho_{i j} \rho_{k l}(a) \underset{\mathrm{def}}{=} \rho_{i j} \otimes \rho_{k l}(\Delta a)=\rho_{k l} \otimes \rho_{i j}(T \circ \Delta a)=\rho_{k l} \otimes \rho_{i j}\left(R \Delta a R^{-1}\right) .
$$

Now use the coalgebra structure (8) to expand the multiplications:

$$
\begin{aligned}
\rho_{i j} \rho_{k l}(a) & =\rho_{k a} \otimes \rho_{i c} \otimes \rho_{a b} \otimes \rho_{c d} \otimes \rho_{b l} \otimes \rho_{d j}\left(R \otimes \Delta a \otimes R^{-1}\right) \\
& =\left(R^{\rho}\right)_{k a, i c} \rho_{a b} \rho_{c d}(a)\left(R^{\rho}\right)_{b l, d j}^{-1},
\end{aligned}
$$

where $R^{\rho}=\rho \otimes \rho(R)$, an $n^{2} \times n^{2}$ matrix of End $(V \otimes V)$. A summation on repeated indices is implied. This simplifies on defining a matrix $\rho$ which is valued in the dual, $\rho \in \operatorname{Mat}\left(n, A^{\prime}\right)$ by $(\rho)_{i j}=\rho_{i j}$. Then in an obvious notation, [7] we obtain:

$$
R^{\rho} \rho_{1} \rho_{2}=\rho_{2} \rho_{1} R^{\rho} \text {. }
$$

The coalgebra can be expressed in the form $\Delta\left(\rho_{1}\right)=\rho_{1} \otimes \rho_{1} \in \operatorname{Mat}\left(n, A^{\prime} \otimes A^{\prime}\right)$, the subscript labelling the endomorphism space.

Consider the case when the Hopf algebra $A$ is the quantised Lie algebra $U_{q} s l(n)$ and the representation $\rho$ is the fundamental representation. From the Peter and Weyl theorem, the fundamental representation generates a dense subspace of the function space Fun $(S l(n))$, with a determinant constraint on the generators. Thus we can define the quantised function space $\operatorname{Fun}_{q}(G l(n))$ as the bialgebra $A(R)$, and $\operatorname{Fun}_{q}(S l(n))$ as the quotient Hopf algebra, defined by introducing a quantum determinant constraint $[6,7,13]$. We denote the generators of $\operatorname{Fun}_{q}(G l(n))$ by $\left\{1, t_{i j}\right\}$, reserving $\rho$ for the representation.

\section{Borel Subalgebras and the Quantum Double}

The Borel Hopf subalgebras of the quantised algebras $U_{q} g$ will be discussed in this section, emphasising the various isomorphisms between the Borel subalgebras and their duals. This analysis introduces the important observation that the Hopf algebra ${ }^{4} U_{q} b_{+}^{\circ}$ used in the construction of the quantum double $D\left(U_{q} b_{+}\right)$, [6] can be realised as the Hopf algebra $U_{q} b_{-}^{\prime}$. This essentially fixes the coalgebra anti-isomorphism $U_{q} b_{+}^{\prime} \rightarrow U_{q} b_{+}^{\circ}$ used in the quantum double construction [6]. Hence the quantum double $D\left(U_{q} b_{+}\right)$is isomorphic, as a $C[[h]]$-module, to the tensor product $U_{q} b_{+} \otimes U_{q} b_{-}^{\prime}$.

First consider the following theorem that relates Hopf subalgebras and biideals ${ }^{5}$ :

\footnotetext{
${ }^{4}$ Here $A^{\circ}$ denotes the dual Hopf algebra $A^{\prime}$ to $A$, with the comultiplication reversed relative to that induced by duality

${ }^{5}$ A multiplicative ideal $I$ that is also a coideal, i.e. $\Delta(I) \subset I \otimes A+A \otimes I$
} 
Theorem 1. Given an Hopf subalgebra B of an Hopf algebra $A$ over the ring $K$, then the annihilator space:

$$
B^{\perp}=\left\{\chi \in A^{\prime}: \chi(B)=0\right\} \subset A^{\prime}
$$

is an ideal and coideal with quotient:

$$
\frac{A^{\prime}}{B^{\perp}} \cong B^{\prime}
$$

Proof. The annihilator space $B^{\perp}$ is obviously a $K$-submodule. The ideal structure of $B^{\perp}$ is considered first. Let $\chi \in B^{\perp}, \zeta \in A^{\prime}$, then:

$$
\chi \cdot \zeta(B)=\chi \otimes \zeta(\Delta B)=0 \text { since } \Delta B \subset B \otimes B .
$$

Hence $\chi \cdot \zeta \in B^{\perp}$. Similarly for the reversed product $\zeta \cdot \chi$. So $B^{\perp}$ is an ideal. It is required to show that for $\chi \in B^{\perp}, \Delta(\chi) \in B^{\perp} \otimes A^{\prime}+A^{\prime} \otimes B^{\perp}$. So it is sufficient to evaluate this on $a \otimes b$, for $a, b \in B$. This is zero as required since $B$ is a subalgebra, i.e. $a \cdot b \in B$. Since $B^{\perp}$ is a biideal, the quotient is well defined as an Hopf algebra. This can be proved to be isomorphic to the dual Hopf algebra $B^{\prime}$ by considering the evaluations on $B$.

The alternative situation where $B$ is an Hopf ideal of $A$ also follows by similar considerations.

This theorem can be applied to the quantised Lie algebras $U_{q} g$. The Hopf subalgebras of most interest are the Borel subalgebras. The Borel subalgebras are denoted $U_{q} b_{ \pm}$, and are generated by $\left\{1, H_{i}, X_{i}^{ \pm}\right\}$respectively. They induce the following quotient structure on the dual:

$$
\begin{aligned}
& U_{q} g^{\prime} \stackrel{\pi^{+}}{\longrightarrow} \frac{U_{q} g^{\prime}}{U_{q} b_{+}^{\perp}} \cong U_{q} b_{+}^{\prime} \cong U_{q} b_{+}, \\
& U_{q} g^{\prime} \stackrel{\pi^{-}}{\longrightarrow} \frac{U_{q} g^{\prime}}{U_{q} b_{-}^{\perp}} \cong U_{q} b_{-}^{\prime} \cong U_{q} b_{-} .
\end{aligned}
$$

This quotient structure implies that if $U_{q} g^{\prime}$ is quasi-triangular, then the Borel subalgebras $U_{q} b_{ \pm}$are also quasi-triangular. However, the Borel subalgebras are only pseudo quasi-triangular, i.e. there is an universal $R$-matrix but it is valued in an embedding Hopf algebra, $U_{q} g$ for example, $[4,6]$. Thus we deduce that $U_{q} g^{\prime}$ is not quasi-triangular. This has important implications in Sect. 3. Similar reasoning applies to the dual of the quantum double, Sect. 7, since the Hopf subalgebras $U_{q} b_{+} \subset D\left(U_{q} b_{+}\right), U_{q} b_{-}^{\prime} \subset D\left(U_{q} b_{+}\right)$induce a similar quotient structure to that in (10).

Consider a general algebra anti-isomorphism, coalgebra isomorphism $\vartheta$ of $U_{q} g$ that interchanges the Borel subalgebras:

$$
\vartheta: U_{q} b_{ \pm} \rightarrow U_{q} b_{\mp} .
$$

For example: $H_{i} \rightarrow H_{i}, X_{i}^{ \pm} \rightarrow X_{i}^{\mp}$. On the dual Hopf algebra, the morphism $\vartheta$ induces a coalgebra anti-isomorphism, thus reversing the comultiplication:

$$
\vartheta^{\prime}: U_{q} g^{\prime} \rightarrow U_{q} g^{\circ}, \quad \vartheta^{\prime}: U_{q} b_{ \pm}^{\perp} \cong U_{q} b_{\mp}^{\perp},
$$


where: $\vartheta^{\prime} \zeta(a)=\zeta(\vartheta a)$ for all $a \in U_{q} g, \zeta \in U_{q} g^{\prime}$. On taking the quotient with $\pi^{ \pm}$we induce an Hopf isomorphism (anti-coalgebra) $\vartheta^{\prime}: U_{q} b_{ \pm}^{\prime} \rightarrow U_{q} b_{\mp}^{\prime}$. Hence $U_{q} b_{+}^{\circ} \cong$ $U_{q} b_{-}^{\prime}$ as Hopf algebras. Thus the two Hopf subalgebras of the quantum double $D\left(U_{q} b_{+}\right)$can be taken to be $U_{q} b_{+}$and $\vartheta^{\prime}\left(U_{q} b_{+}^{\prime}\right)=U_{q} b_{-}^{\prime}$. Hence, introducing the dual bases $\left\{\zeta_{s}\right\} \in U_{q} b_{+},\left\{\eta^{s}\right\} \in U_{q} b_{+}^{\prime}$, a basis for the quantum double $D\left(U_{q} b_{+}\right)$is $\left\{\zeta_{s} \otimes \vartheta^{\prime}\left(\eta^{t}\right)\right\}$, [6] with the universal $R$-matrix given by the canonical element of $U_{q} b_{+} \otimes U_{q} b_{-}^{\prime},[6]:$

$$
R=\sum_{s} \zeta_{s} \otimes \vartheta^{\prime}\left(\eta^{s}\right)
$$

The inverse of the universal $R$-matrix is, [4]:

$$
R^{-1}=S \otimes 1(R)=1 \otimes S_{\circ}(R)
$$

The symbols $S$ and $S_{\text {。 }}$ denote the antipode and skew-antipode respectively [1].

In the rest of this paper, the two maps $\vartheta, \vartheta^{\prime}$ will be understood as restricted to the Borel subalgebras:

$$
\vartheta: U_{q} b_{-} \rightarrow U_{q} b_{+}, \quad \vartheta^{\prime}: U_{q} b_{+}^{\prime} \rightarrow U_{q} b_{-}^{\prime} .
$$

Recall that the universal $R$-matrix for $U_{q} g$ is obtained via a quotient mapping $\pi: D\left(U_{q} b_{+}\right) \rightarrow U_{q} g$ [6], that can be taken to satisfy $\left.\pi\right|_{U_{q} b_{+}}=1$ [4]. The resultant $R$-matrix for $U_{q} g$ is independent of the isomorphism $\vartheta^{\prime}$, a change in $\vartheta^{\prime}$ being compensated by a change in the quotient map $\pi$.

\section{Representations of the Dual $U_{q} g^{\prime}$}

It is desired to reformulate the Hopf structure of the quantised algebra $U_{q} g$ into a matrix form similar to that achieved for the quantum group in Sect. 1. Hence a representation of the quantum group $U_{q} g^{\prime}$ is required. This guarantees the coalgebra relation (8). However the formulation of the algebra into a matrix equation similar to (9) cannot be accomplished until an universal $R$-matrix is introduced. Since $U_{q} g^{\prime}$ does not possess an $R$-matrix, Sect. 2, it is necessary to find an homomorphism of $U_{q} g^{\prime}$ into a quasi-triangular Hopf algebra. The chosen algebra is $U_{q} g$, this giving us compatibility with the matrix formulation of the quantum group $U_{q} g^{\prime}$ in Sect. 1 .

Given a representation $\rho: U_{q} g \rightarrow \operatorname{End}(V, K)$ we can construct a representation of $U_{q} g^{\prime}$ (more strictly a representation of the QUE algebra equivalent, [6]) by taking the quotient to the Hopf algebras of (10) and using representations of $U_{q} b_{ \pm}$. However, since the Hopf algebras $U_{q} b_{ \pm}$are only pseudo-quasi-triangular, [4] it is necessary to use a representation of $U_{q} g$ in which the Hopf algebras $U_{q} b_{ \pm}$are embedded, this giving access to an universal $R$-matrix. We define the two representations $\sigma^{ \pm}=\rho^{\circ} \phi^{ \pm} \circ \pi^{ \pm}$of $U_{q} g^{\prime}$. Here $\phi^{ \pm}$are algebra homomorphisms of $U_{q} b_{ \pm}^{\prime}$ into $U_{q} g$, with coalgebra properties to be determined. As before the individual matrix elements are elements of the dual, i.e. $\sigma_{i j}^{ \pm} \in U_{q} g$. Since the maps $\sigma^{ \pm}$are representations we have the coalgebra structure (compare to (8)):

$$
\Delta \sigma_{i j}^{ \pm}=\sum_{k} \sigma_{i k}^{ \pm} \otimes \sigma_{k j}^{ \pm}
$$


To define the homomorphisms $\phi^{ \pm}$, we observe that the generators $\sigma_{i j}^{ \pm} \in U_{q} g$ can be expressed in a form reminiscent of the universal $R$-matrix of $U_{q} g$ :

$$
\begin{aligned}
\sigma_{i j}^{ \pm}=\sum_{s} \xi_{s}\left(\xi^{s}, \sigma_{i j}^{ \pm}\right) & =\sum_{\xi_{s} \in U_{q} b_{ \pm}} \xi_{s} \rho_{i j}{ }^{\circ} \phi^{ \pm} \circ \pi^{ \pm}\left(\xi^{s}\right) \\
& =\operatorname{Id} \otimes \rho_{i j} \circ \phi^{ \pm}\left(\sum_{\xi_{s \in U_{q} b_{ \pm}}} \xi_{s} \otimes \pi^{ \pm} \xi^{s}\right) .
\end{aligned}
$$

Here the following dual bases have been defined:

$$
\left\{\xi_{s}\right\} \in U_{q} g, \quad\left\{\xi^{s}\right\} \in U_{q} g^{\prime},
$$

for the index $s$ in some suitable index set.

The projection operator $\pi^{ \pm}$reduces the sum over the basis of $U_{q} g^{\prime}$ to that over $\operatorname{Im}\left(\pi^{ \pm}\right)=U_{q} b_{ \pm}^{\prime}$, implying that $\sigma_{i j}^{ \pm} \in U_{q} b_{ \pm}$. In this expression the paired elements are dual: $\pi^{ \pm} \xi^{s}\left(\xi_{t}\right)=\delta_{t^{s}}^{s}$. Thus the term in brackets is very similar to the universal $R$-matrix of $D\left(U_{q} b_{+}\right)$, (11). The representations are chosen to maximise this identification. If we choose $\phi^{+}=\pi \circ \vartheta^{\prime}$, we obtain from (11), (14):

$$
\sigma_{i j}^{+}=\operatorname{Id} \otimes \rho_{i j}\left(R_{U_{q} g}\right) \in U_{q} b_{+} .
$$

Hence the representation $\phi^{+}$is equivalent to the sequence of maps:

$$
U_{q} g^{\prime} \stackrel{R}{\longrightarrow} U_{q} g \stackrel{\rho}{\longrightarrow} \operatorname{End}(V, K),
$$

where the first map is given by evaluation on the first position of $R$.

The second representation $\sigma^{-}$is more difficult. It involves the algebra anti-homomorphism $\vartheta$ as follows:

$$
U_{q} g^{\prime} \stackrel{\pi^{-}}{\longrightarrow} U_{q} b_{-}^{\prime} \stackrel{\text { S॰g }}{\longrightarrow} U_{q} b_{+} \stackrel{1}{\longrightarrow} U_{q} g \stackrel{\rho}{\longrightarrow} \operatorname{End}(V, K)
$$

Fig. 1

i.e. $\phi^{-}=\pi \circ S \circ \vartheta$. The Hopf algebra $U_{q} b_{+}$has been embedded in the quantum double, which allows us to use the quotient mapping $\pi: D\left(U_{q} b_{+}\right) \rightarrow U_{q} g$.This leads to the identity mapping in Fig. 1 since $\left.\pi\right|_{U_{q} b_{+}}=1$ [4]. The antipode has to be included to obtain an algebra homomorphism. The only other canonical anti-algebra homomorphism is the skew antipode. It is observed that $\sigma^{-}$is defined with the algebra anti-homomorphism $\vartheta(13)$ acting on the quotient Hopf algebra $U_{q} b_{-}^{\prime}(10)$. However this Hopf algebra is isomorphic to $U_{q} b_{-}$. In fact, by using the dual to the quantum double, Sect. 7 the roles of $U_{q} b_{-}$and $U_{q} b_{-}^{\prime}$ are interchanged.

Since $\left(\vartheta^{-1} \xi_{s}, \vartheta \xi^{t}\right)=\left(\xi_{s}, \xi^{t}\right)=\delta_{s}^{t}$, the matrix of generators $\sigma^{-}$can be verified to be (using (12), (14)):

$$
\sigma_{i j}^{-}=\rho_{i j} \otimes \operatorname{Id}\left(R_{U_{q} g}^{-1}\right) \equiv \rho_{i j} \otimes S_{\circ}\left(R_{U_{q} g}\right) \in U_{q} b_{-} .
$$

Note that the evaluation structure between the matrices $t$ and $\sigma$ is that of [7]:

$$
\left(t, \sigma^{+}\right)=R^{\rho}, \quad\left(\sigma^{-}, t\right)=R^{\rho-1} .
$$


The two homomorphisms $\sigma^{ \pm}$have now been constructed, with the embedding homomorphisms:

$$
\phi^{+}=\pi \circ \vartheta^{\prime}, \quad \phi^{-}=\pi \circ S \circ \vartheta .
$$

Note that both $\phi^{ \pm}$are coalgebra anti-homomorphisms since they contain $\vartheta^{\prime}$ and the antipode $S$ respectively. The various homomorphisms can be summarised in the (non-commuting) diagram:

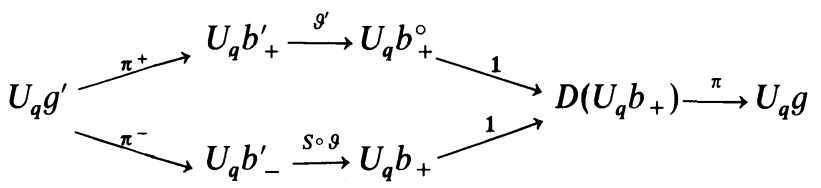

Fig. 2

All the maps are available from the quantum double construction. The map $\vartheta$ is arbitrary, it is only required to be an anti-algebra isomorphism and coalgebra isomorphism such that it exchanges the Borel subalgebras $9: U_{q} b_{ \pm} \rightarrow U_{q} b_{\mp}$. The above combinations of maps are independent of $\vartheta$ up to the arbitrary isomorphism $\left.\pi\right|_{U_{q} b_{+}}$. This is proved in Sect. 7. This can also be deduced from the expressions (15), (16). Note that since $U_{q} g^{\prime}$ is not isomorphic to $U_{q} g$, any Hopf homomorphism $U_{q} g^{\prime} \rightarrow U_{q} g$ has a non-zero kernel, which is a biideal. The possible biideals correspond to the following Hopf subalgebras of $U_{q} g: U_{q} h, U_{q} \tilde{g}$ ( $\tilde{g}$ subalgebra of $g$ ) and the Borel subalgebras (of $g$ and $\tilde{g}$ ). The Borel subalgebras $U_{q} b_{ \pm}$produce the maximal subalgebras of $U_{q} g$ that can be generated by the above construction involving representations.

The multiplication structure satisfied by the generators $\sigma^{ \pm}$can now be deduced. We can proceed in two ways. Using the definitions (15) and (16) in terms of the universal $R$-matrix, we can use the QYBE equation to derive (6). This is carried out in [3]. Alternatively these can be derived totally in terms of the representation structure of $\sigma^{ \pm}$. This course is pursued here. Hence by duality we have:

$$
\left(\sigma_{i j}^{ \pm} \sigma_{k l}^{ \pm}, \zeta\right)=\left(\sigma_{i j}^{ \pm} \otimes \sigma_{k l}^{ \pm}, \Delta \zeta\right)=\left(\rho_{i j} \otimes \rho_{k l}, T \circ \Delta\left(\phi^{ \pm} \circ \pi^{ \pm} \zeta\right)\right) \quad \forall \zeta \in U_{q} g^{\prime} .
$$

Since $\phi^{ \pm} \circ \pi^{ \pm} \zeta \in U_{q} g$, the existence of an $R$-matrix may now be used to deduce a matrix form of the algebra structure. The calculation is identical to that in Sect. 1, with the inverse of the $R$-matrix occurring where the $R$-matrix was used before. The Hopf structure of the generators $\sigma_{i j}^{ \pm} \in U_{q} b_{ \pm}$can now be expressed as:

$$
\Delta \sigma_{i j}^{ \pm}=\sum_{k} \sigma_{i k}^{ \pm} \otimes \sigma_{k j}^{ \pm}, R_{21}^{\rho} \sigma_{1}^{ \pm} \sigma_{2}^{ \pm}=\sigma_{2}^{ \pm} \sigma_{1}^{ \pm} R_{21}^{\rho} .
$$

If the fundamental representation of $U_{q} s l(n)$ is used, then the Borel subalgebras $U_{q} b_{ \pm}$are generated $^{6}$ by $\left\{1, \sigma_{i j}^{ \pm}\right\}$. This follows from (15), (16) since the fundamental representation is faithful on the Chevalley generators, and the universal $R$-matrix of $D\left(U_{q} b_{+}\right)$is the canonical element of $U_{q} b_{+} \otimes U_{q} b_{-}^{\prime}$, [6]. The Borel subalgebras

\footnotetext{
${ }^{6}$ As defined by Jimbo [10], i.e. only the combination $k_{i}=q^{H_{i / 2}}$ occurring
} 
are generated as QUE algebras [6], since in the classical limit $\sigma^{ \pm} \rightarrow I+h \eta^{ \pm}$, where $\eta^{ \pm}$are matrices of generators for the Lie algebra $s l(n)$.

The commutation relations between the two sets of generators $\sigma_{i j}^{ \pm}$still has to be derived. These are more difficult than the calculation of those within the two sets, and their form depends upon the choice of the representations $\sigma^{ \pm}=\rho^{\circ} \phi^{ \pm} \circ \pi^{ \pm}$. Compare with the previous result where $R_{21}^{\rho} \sigma_{1}^{ \pm} \sigma_{2}^{ \pm}=\sigma_{2}^{ \pm} \sigma_{1}^{ \pm} R_{21}^{\rho}$ depends only on the morphisms $\phi^{ \pm}$(18) being algebra homomorphisms, coalgebra antihomomorphisms. The following result is proved in Sect. 7:

$$
R_{21}^{\rho} \sigma_{1}^{+} \sigma_{2}^{-}=\sigma_{2}^{-} \sigma_{1}^{+} R_{21}^{\rho} \text {, where } R_{21}^{\rho}=\rho \otimes \rho(T \circ R) .
$$

The equations in (19), (20) are the Hopf structure given in [7], for $U(R)(6)$. The duality structure in (5) is also reproduced from (17) and the coalgebra relations in (19).

\section{Quantum Determinants and Constraints on Generators}

In this section we restrict our analysis to the quantised algebras $U_{q} s l(n)$ with $\rho$ its fundamental representation (42). We consider the definitions of the generators $\left\{t_{i j}\right\}$ and $\left\{\sigma_{i j}^{ \pm}\right\}$, Sect. 1, (15), (16) and the constraints on these generators that are imposed due to the form of the universal $R$-matrix and the quantum determinant condition on the representation $\rho$. The occurrence of the constraints in the quantised function approach of [7] through the definition (5) is also considered, ultimately being a consequence of the algebraic relations satisfied by the $R$-matrix in the fundamental representation, (23). The determinant constraint on the quantum group must be imposed to obtain the correct duality. For the fundamental representation (42), $\rho$ satisfies no further conditions; hence $A(R)$ and $U(R)$ are dual, [7].

The diagonal parts of $\sigma^{+}$and $\sigma^{-}$are only affected by the coroot prefactor of the universal $R$-matrix; this having a form $[6,2,14]$ :

$$
\exp \left(\frac{h}{2} \sum_{i j} a_{i j}^{-1} H_{i} \otimes H_{j}\right) \text {. }
$$

Since the diagonal parts (15), (16) only differ by an antipodal action, we obtain the constraint [7]: $\sigma_{i i}^{-}=\sigma_{i i}^{+-1}, i \in[1, n]$, since $S\left(H_{i}\right)=-H_{i}$.

All other constraints are consequences of the quantum determinant condition satisfied by the representation $\rho$. The quantum determinant can be expressed in terms of a quantum Levi-Civita symbol:

$$
\varepsilon_{i j k \ldots}^{q}= \begin{cases}\left(-q^{-1}\right)^{l(i, j, k . .)} & \{i, j, . .\} \in S_{n} \\ 0 & \text { otherwise. }\end{cases}
$$

Here $\{i j k .$.$\} denotes a permutation of the integers 1 \ldots n, S_{n}$ the permutation group on $n$ objects, and $l(i, j, k .$.$) the length of the permutation, i.e. the number of inversions$ to reach $\{1,2,3 .$.$\} . For example, if n=3$ then $l(3,2,1)=3$.

The quantum determinant is expressed in the form:

$$
\varepsilon_{i_{1} i_{2} \ldots i_{n}}^{q} t_{j_{1} i_{1}} t_{j_{2} i_{2}} \ldots t_{j_{n} i_{n}}=\varepsilon_{j_{1} j_{2} \ldots j_{n}}^{q} \operatorname{det}_{q}(t)
$$


or

$$
t_{1} t_{2} \ldots t_{n} \Omega=\operatorname{det}_{q}(t) \Omega
$$

by defining a vector $\Omega$ in $V^{\otimes(n)}: \Omega_{i_{1} i_{2} \ldots i_{n}}=\varepsilon_{i_{1} i_{2} \ldots i_{n}}^{q}$. We note that this definition for the quantum determinant is specific to the Hopf structure in (4) with the $U_{q} s l(n)$ $R$-matrix in the fundamental representation, (42), i.e. $R$-matrix (51). The appropriate definition for other representations and quantum groups are most easily treated using the comodule structures of $[12,13]$. These will not be discussed.

Consider the bialgebra $\operatorname{Fun}_{q}(G l(n))$ generated by $\left\{1, t_{i j}\right\}$ satisfying (4) with the matrix $R$ in the fundamental representation of $U_{q} s l(n)$. Then the quantum determinant, as defined by (21), has the properties:

1. Group like [1]:

$$
\Delta\left(\operatorname{det}_{q}(t)\right)=\operatorname{det}_{q}(t) \otimes \operatorname{det}_{q}(t) \in \operatorname{Fun}_{q}(G l(n)) \otimes \operatorname{Fun}_{q}(G l(n)) .
$$

2. Multiplicative, for commuting generators; $s_{1} t_{2}=t_{2} s_{1}$ :

$$
\operatorname{det}_{q}(s \cdot t)=\operatorname{det}_{q}(s) \cdot \operatorname{det}_{q}(t) \in \operatorname{Fun}_{q}(G l(n)) \otimes \operatorname{Fun}_{q}(G l(n)) \text {. }
$$

3. Invariant under transposition:

$$
\operatorname{det}_{q}\left(t^{T}\right)=\operatorname{det}_{q}(t)
$$

4. Lies in the centre of the bialgebra $\operatorname{Fun}_{q}(G l(n))$, i.e.:

$$
\left[\operatorname{det}_{q}(t), t_{i j}\right]=0 \text {. }
$$

Note that the matrices $s \cdot t$ and $t^{T}$ satisfy Eq. (4) as required for the quantum determinant to be well defined. Properties 1 through 3 follow from the definition (21). Property 4 is a consequence of the quantum determinant condition on the representation $\rho$ used for the $R$-matrix in (4):

$$
\operatorname{det}_{q}(\rho)=1 \text {. }
$$

This is the quantum generalisation of the determinant condition for a representation of $S l(n)$. Note that $\rho$ satisfies the algebra (4), Sect. 1. Equation (22) follows from observation [6], using (21), (42).

Relation (22) introduces four algebraic relations on the $R$-matrix in the fundamental representation. For example, consider the combination:

Or:

$$
\begin{aligned}
R_{1, n+1} R_{2, n+1} \ldots R_{n, n+1} \Omega_{1 \ldots . n} & =\rho^{\otimes(n)} \Omega_{1 \ldots n} \otimes \rho\left(\Delta^{(n-1)} \otimes \operatorname{Id}\left(R_{U_{q} s l(n)}\right)\right) \\
& =\Omega_{1 \ldots . n} \operatorname{det}_{q}(\rho) \otimes \rho\left(R_{U_{q} s l(n)}\right)=\Omega_{1 \ldots n} I_{n+1} .
\end{aligned}
$$

$$
\varepsilon_{i_{1} i_{2} \ldots i_{n}}^{q} R_{j_{1} i_{1}, k_{1} k_{2}} R_{j_{2} i_{2}, k_{2} k_{3}} \ldots R_{j_{n-1} i_{n}, k_{n} k_{n+1}}=\varepsilon_{j_{1} j_{2} \ldots j_{n}}^{q} \delta_{k_{1} k_{n+1}} .
$$

Here we have used the duality between multiplication in $U_{q} s l(n)^{\prime}$ and comultiplication in $U_{q} s l(n)$, and the quasi-triangular and counit properties of the universal $R$-matrix [6]:

$$
\Delta \otimes \operatorname{Id}(R)=R^{13} R^{23}, \quad \varepsilon \otimes \operatorname{Id}(R)=1 .
$$

There are three similar equations with contraction of the $\varepsilon$ tensor on other indices. 
All the determinant constraints of [7] are consequences of these algebraic relations and definition (5).

Consider the commutation of the quantum determinant with the $t$ generators:

$$
\begin{aligned}
\operatorname{det}_{q}(t) t_{n+1} \Omega_{1 . . n}= & t_{1} t_{2} \ldots t_{n} t_{n+1} \Omega_{1 . . n} \\
= & \left(R_{1, n+1} R_{2, n+1} \ldots R_{n, n+1}\right)^{-1} t_{n+1} t_{1} t_{2} \ldots t_{n} \\
& \cdot\left(R_{1, n+1} R_{2, n+1} \ldots R_{n, n+1}\right) \Omega_{1 . . n} \\
= & t_{n+1} \operatorname{det}_{q}(t) \Omega_{1 . . n} .
\end{aligned}
$$

The second line follows on using the algebra (4), and the last line from the eigenvector equation (23). Hence we deduce the commutivity: $\left[\operatorname{det}_{q}(t), t_{i j}\right]=0$. Since $\operatorname{det}_{q}(t)$ is also group-like, $\operatorname{det}_{q}(t)-1$ defines a biideal, and hence the quotient is well defined as an Hopf algebra. This is $\operatorname{Fun}_{q}(\operatorname{Sl}(n))$. The necessity for taking this quotient in the work of Faddeev et al. [7] is a consequence of the evaluation structure in (5), and the algebraic relation (23):

$$
\begin{aligned}
\left(\operatorname{det}_{q}(t) \Omega_{1 \ldots n}, \sigma_{n+1}^{+}\right) & =\left(t_{1} t_{2} \ldots t_{n} \Omega_{1 \ldots n}, \sigma_{n+1}^{+}\right) \\
& =R_{1, n+1} R_{2, n+1} \ldots R_{n, n+1} \Omega_{1 . . n}=\Omega_{1 . . n} I_{n+1} .
\end{aligned}
$$

Hence $\left(\operatorname{det}_{q}(t)-1, \sigma^{+}\right)=0$. Similarly for $\sigma^{-}$. Thus we must set $\operatorname{det}_{q}(t)=1$ to obtain the correct duality structure. Similarly for the quantum determinant constraints on $\sigma^{ \pm}$:

$$
\begin{aligned}
\left(\operatorname{det}_{q^{-1}}\left(\sigma^{+}\right) \Omega_{1 \ldots n}^{q^{-1}}, t_{n+1} t_{n+2} \ldots t_{n+k}\right) \\
\quad=\left(\sigma_{1}^{+} \sigma_{2}^{+} \ldots \sigma_{n}^{+} \Omega_{1 \ldots n}^{q^{-1}}, t_{n+1} t_{n+2} \ldots t_{n+k}\right) \\
\quad=\left(\sigma_{1}^{+} \otimes \sigma_{2}^{+} \otimes \ldots \otimes \sigma_{n}^{+}, \Delta^{(n-1)}\left(t_{n+1} t_{n+2} \ldots t_{n+k}\right)\right) \Omega_{1 \ldots n}^{q^{-1}} \\
=\left(\sigma_{1}^{+}, t_{n+1} \ldots t_{n+k}\right)\left(\sigma_{2}^{+}, t_{n+1} \ldots t_{n+k}\right) \ldots\left(\sigma_{n}^{+}, t_{n+1} \ldots t_{n+k}\right) \Omega_{1 \ldots n}^{q^{-1}} \\
=\left(R_{n+1,1} R_{n+1,2} \ldots R_{n+1, n}\right) \ldots\left(R_{n+k, 1} R_{n+k, 2} \ldots R_{n+k, n}\right) \Omega_{1 \ldots n}^{q^{-1}} \\
=\Omega_{1 \ldots n}^{q^{-1}} I_{n+1 \ldots n+k} .
\end{aligned}
$$

Similarly for the matrix $\sigma^{-}$. Hence definition (5) imposes the determinant constraints. In contrast, these determinant constraints are transparent from the viewpoint of our construction, since the generators $\left\{t_{i j}\right\}$ and $\left\{\sigma_{i j}^{ \pm}\right\}$are defined in terms of the representation $\rho$, Sect. $1,(15),(16)$ respectively. Since $\left\{t_{i j}\right\}$ are defined directly from $\rho$, we obtain $\operatorname{det}_{q}(t)=1$. By definition $\sigma^{ \pm}=\rho^{\circ} \phi^{ \pm} \circ \pi^{ \pm}$; hence we deduce:

$$
\begin{aligned}
\left(\operatorname{det}_{q^{-1}}\left(\sigma^{ \pm}\right), a\right) & =\sum_{\left\{i_{s}\right\} \in S_{n}}(-q)^{1\left(\left\{i_{s}\right\}\right)}\left(\rho_{1 i_{1}} \otimes \rho_{2 i_{2}} \otimes \ldots \otimes \rho_{n i_{n}}, \quad\left(\phi^{ \pm} \circ \pi^{ \pm}\right)^{\otimes(n)} \Delta^{(n-1)}(a)\right) \\
& =\left(\sum_{\left\{i_{s}\right\} \in S_{n}}(-q)^{1\left(\left(i_{s}\right\}\right)} \rho_{n i_{n}} \rho_{n-1 i_{n}-1} \ldots \rho_{1 i_{1}}, \phi^{ \pm} \circ \pi^{ \pm}(a)\right) \\
& =\left(\operatorname{det}_{q}(\rho), \phi^{ \pm} \circ \pi^{ \pm}(a)\right), \quad \forall a \in U_{q} g^{\prime} .
\end{aligned}
$$

Hence:

$$
\operatorname{det}_{q^{-1}}\left(\sigma^{ \pm}\right)=\prod_{i=1}^{n} \sigma_{i i}^{ \pm}=1
$$


using in the second expression the fact that $\sigma^{ \pm}$are triangular (valid for a representation taking the Borel subalgebras to triangular matrices). Notice that the determinant is defined with a $q^{-1}$ relative to (21). This is consistent with the differences in the algebra relations in (9), (19) since $R^{\rho}(q)^{-1}=R^{\rho}\left(q^{-1}\right)$.

Finally, note that the antipode is intimately related to the determinant constraints, an antipodal mapping not existing in $\mathrm{Fun}_{q}(G l(n))$. However $\mathrm{Fun}_{q}(S l(n))$ has an antipode, since the dual is an Hopf algebra. In the matrix format, the antipode is defined by [7]:

$$
S(t) \cdot t=t \cdot S(t)=I
$$

This implies ${ }^{7}$ that $\operatorname{det}_{q}(t)^{-1}=\operatorname{det}_{q}(S(t))$. However since the only invertible element in $\operatorname{Fun}_{q}(G l(n))$ is the identity, we must quotient by $\operatorname{det}_{q}(t)=1$ if an antipode is to exist. Equation (25) also implies that $S(t)=t_{c o}^{T}$, a relation given in [7], where:

$$
\begin{aligned}
\left(t_{c o}\right)_{k j} & =(-q)^{k-1} \varepsilon_{j i_{1} . . i_{m-1}}^{q} t_{1 i_{1}} \ldots t_{k-1 i_{k-1}} t_{k+1 i_{k}} \ldots t_{m i_{m-1}} \\
& =(-q)^{k-j} \operatorname{det}_{q} t_{\hat{k} \hat{j}} .
\end{aligned}
$$

Here $t_{\hat{k} \hat{j}}$ denotes the comatrix of $t$, i.e. $t$ with row $k$ and column $j$ removed.

\section{Borel Structure Isomorphisms}

In this section the matrix format (6) of the Hopf structure of the quantised algebra $U_{q} s l(n)$ will be discussed, demonstrating the incorporation of the Borel structure of $U_{q} s l(n)$ and the quotient structure of $U_{q} s l(n)^{\prime}$ in the matrix formulation. The representation is assumed to take the Borel subalgebras $U_{q} b_{ \pm}$to upper and lower triangular matrices respectively.

The generators $\sigma_{i j}^{+}, \sigma_{i j}^{-}$generate isomorphic subalgebras. This is demonstrated by matrix transposition. Since $\sigma^{+}$and $\sigma^{-}$are lower and upper triangular, transposing is expected to produce some type of isomorphism. We have $R_{12}^{T_{1} T_{2}}=R_{21}$, where $T_{i}$ is transposition in position $i$; hence:

or

$$
\left(R_{21} \sigma_{1}^{-} \sigma_{2}^{-}\right)^{T_{1} T_{2}}=\sigma_{1}^{-T} \sigma_{2}^{-T} R_{12}=R_{12} \sigma_{2}^{-T} \sigma_{1}^{-T}
$$

$$
R_{21} \sigma_{1}^{-T} \sigma_{2}^{-T}=\sigma_{2}^{-T} \sigma_{1}^{-T} R_{21} \text {. }
$$

Since the determinant conditions, (24) are also invariant under transposition, matrix transposition induces an algebra isomorphism $\sigma^{-T} \rightarrow \sigma^{+}$. By considering the effect of transposing the coalgebra relation $\Delta \sigma_{i j}^{-}=\sum_{k} \sigma_{i k}^{-} \otimes \sigma_{k j}^{-}$, it can be proved that this is also a coalgebra anti-isomorphism. Due to this isomorphism, only the commutation relations for $\sigma^{+}$need to be evaluated. The algebra relation $R_{21} \sigma_{1}^{+} \sigma_{2}^{-}=\sigma_{2}^{-} \sigma_{1}^{+} R_{21}$ is invariant under matrix transposition; hence the commutation relations must be invariant under the transformation of generators implied by $\sigma^{-T} \leftrightarrow \sigma^{+}$. This transformation corresponds to the algebra isomorphism, coalgebra anti-isomorphism $H_{i} \rightarrow-H_{i}, X_{i}^{ \pm} \rightarrow-q^{ \pm 1} X_{i}^{\mp}$.

${ }^{7}$ A proof of this fact is not as obvious as it would appear from (25), since $S(t)$ and $t$ do not commute 
By Theorem 1, we know that the Borel subalgebras induce a quotient structure on the quantum group, as given in (10). This structure can be demonstrated in the matrix format. The strictly lower triangular terms of the $t$ matrix generate the biideal $U_{q} b_{+}^{\perp}$ (valid for any representation taking $U_{q} b_{+}$to upper triangular matrices). The biideal property can also be deduced from the form of the $R$-matrix (51), and the coalgebra relation in (4). Taking the quotient with $U_{q} b_{+}^{\perp}$ sets the strictly lower triangular terms to zero. By transposing and acting with the antipode, (or skew antipode) we obtain the $\sigma^{+}$Hopf structure (6), i.e. $U_{q} b_{+}^{\prime}, U_{q} b_{+}$are isomorphic as Hopf algebras (the homomorphism involves two coalgebra antihomomorphisms). Similar reasoning applies to the strictly upper triangular terms.

\section{An Example: $U_{q} s l(2)$}

The quantised algebra $U_{q} s l(2)$ is generated by the generators $\left\{1, H, X^{+}, X^{-}\right\}$with the Hopf structure given in (2). In this example we shall construct the quantum group and express the commutation relations of $U_{q} s l(2)$ in the matrix form (6). This involves constructing the matrices $\sigma^{ \pm} \in \operatorname{Mat}\left(n, U_{q} b_{ \pm}\right)$(15), (16). The fundamental representation will be used [6]:

$$
\rho(H)=\left(\begin{array}{rr}
1 & 0 \\
0 & -1
\end{array}\right), \quad \rho\left(X^{+}\right)=\left(\begin{array}{ll}
0 & 1 \\
0 & 0
\end{array}\right), \quad \rho\left(X^{-}\right)=\left(\begin{array}{ll}
0 & 0 \\
1 & 0
\end{array}\right) .
$$

The universal $R$-matrix has a form [6]:

$$
R_{U_{q} s(2)}=\exp \left(\frac{h}{4} H \otimes H\right)\left(1+\left(1-q^{-2}\right) q^{H / 2} X^{+} \otimes q^{-H / 2} X^{-}+\ldots . .\right) .
$$

The dots indicate irrelevant terms since they project to zero under the representation; $\rho\left(X^{ \pm n}\right)=0$ for $n>1$. The universal $R$-matrix in this representation can be proved to be:

$$
R^{\rho}=q^{-1 / 2}\left(\begin{array}{cccc}
q & 0 & 0 & 0 \\
0 & 1 & \left(q-q^{-1}\right) & 0 \\
0 & 0 & 1 & 0 \\
0 & 0 & 0 & q
\end{array}\right)
$$

This differs from the expression in [7] due to a different choice of representation, see (29). From Sect. 1 we have the following Hopf structure for the generators $t_{i j}$ of the quantum group:

$$
R^{\rho} t_{1} t_{2}=t_{2} t_{1} R^{\rho}, \quad \Delta t_{i j}=\sum_{k} t_{i k} \otimes t_{k j}
$$

Expressing the $t$ matrix as: $t=\left(\begin{array}{cc}k & W^{+} \\ W^{-} & k^{\prime}\end{array}\right)$, the Hopf structure reads:

$$
\begin{aligned}
{\left[k^{\prime}, k\right] } & =\left(q-q^{-1}\right) W^{+} W^{-}, \\
k W^{ \pm} & =q^{-1} W^{ \pm} k, \quad k^{\prime} W^{ \pm}=q W^{ \pm} k^{\prime}, \\
W^{+} W^{-} & =W^{-} W^{+} .
\end{aligned}
$$




$$
\begin{aligned}
\Delta k & =k \otimes k+W^{+} \otimes W^{-}, \\
\Delta k^{\prime} & =k^{\prime} \otimes k^{\prime}+W^{-} \otimes W^{+}, \\
\Delta W^{+} & =W^{+} \otimes k^{\prime}+k \otimes W^{+}, \\
\Delta W^{-} & =W^{-} \otimes k+k^{\prime} \otimes W^{-} .
\end{aligned}
$$

Compare this to the $U_{q} s l(2)$ Hopf structure in (2). The quantised function space $\operatorname{Fun}_{q}(G l(2))$ is generated by $\left\{1, t_{i j}\right\}$, and the quantum determinant constraint quotients this to the Hopf algebra $\operatorname{Fun}_{q}(\operatorname{Sl}(2))$ : $\operatorname{det}_{q}(t)=k k^{\prime}-q^{-1} W^{+} W^{-}=1$.

The Hopf ideals $U_{q} b_{ \pm}^{\perp}$ can now be constructed. Observe that the following elements generate the Borel ideals:

$$
\begin{aligned}
& k \cdot k^{\prime}-1, k^{\prime} \cdot k-1, W^{-} \in U_{q} b_{+}^{\perp}, \\
& k \cdot k^{\prime}-1, k^{\prime} \cdot k-1, W^{+} \in U_{q} b_{-}^{\perp} .
\end{aligned}
$$

Hence the following quotient structure is obtained for $U_{q} b_{+}^{\prime}$ :

$$
\begin{aligned}
k^{\prime} & =k^{-1}, \\
k W^{+} & =q^{-1} W^{+} k, \\
\Delta k & =k \otimes k, \\
\Delta W^{+} & =W^{+} \otimes k^{-1}+k \otimes W^{+} .
\end{aligned}
$$

This is isomorphic to the Hopf subalgebra $U_{q} b_{+}$under the identification $k=q^{-H / 2}$, $W^{+}=X^{+}$. The combination $k$ occurs instead of $H$, corresponding to the quantisation of the Lie algebra $s l(2)$ as defined in [9]. This isomorphism is transposition and antipodal action as discussed in Sect. 5. A similar analysis holds for $U_{q} b_{-}^{\prime}$.

The matrices $\sigma^{ \pm}$will now be constructed from the definitions (15), (16). Using the universal $R$-matrix in (26), the following can be verified:

$$
\sigma^{+}=\left(\begin{array}{cc}
q^{H / 2} & 0 \\
q^{-1 / 2}\left(q-q^{-1}\right) X^{+} & q^{-H / 2}
\end{array}\right), \quad \sigma^{-}=\left(\begin{array}{cc}
q^{-H / 2} & -q^{1 / 2}\left(q-q^{-1}\right) X^{-} \\
0 & q^{H / 2}
\end{array}\right) .
$$

The Hopf structure of $U_{q} s l(2)$ can be reproduced from the formulae (6), using (27). Observe that only the combination $q^{H / 2}$ occurs and not $H$ itself. It is interesting to observe that with this combination it is unnecessary to go to the enveloping algebra, since the commutation relations close. This is also true of $U_{q} s l(3)$, but not for the higher dimensional Lie algebras $U_{q} s l(n)$. This is due to the commutation relation, (52):

$$
\operatorname{ad}_{q} e_{\alpha} \cdot e_{\beta}=e_{\alpha} e_{\beta}-e_{\beta} e_{\alpha}=\left(q-q^{-1}\right) e_{\gamma} e_{\bar{\alpha}+\beta},
$$

which produces a product of generators. In this commutation relation the roots $\alpha, \beta$ are such that $\alpha+\beta$ is not a root and there exists a non-zero overlap of simple roots in $\alpha, \beta$ and no inclusion. Hence three or more simple roots are required. The notation is further explained in Sect. 8.

Instead of the fundamental representation $\rho$, any other representation may be used. Of greatest interest is the representation $\rho^{\prime}$ defined by:

$$
\rho^{\prime}(H)=\left(\begin{array}{rr}
-1 & 0 \\
0 & 1
\end{array}\right), \quad \rho^{\prime}\left(X^{+}\right)=\left(\begin{array}{ll}
0 & 0 \\
\alpha & 0
\end{array}\right), \quad \rho^{\prime}\left(X^{-}\right)=\left(\begin{array}{cc}
0 & \alpha^{-1} \\
0 & 0
\end{array}\right),
$$


where alpha is an arbitrary constant. This is related to the fundamental representation by $\rho^{\prime}=\rho^{T} \circ S_{\circ}$ for the specific value $\alpha=-q^{-1}$. Notice that two algebra anti-homomorphisms are involved, transposition and the skew-antipode. A direct calculation of $\sigma^{ \pm}$is unnecessary since the results for $\rho$ can be employed:

$$
\sigma^{+^{\prime}}=1 \otimes \rho^{T} \circ S_{\circ}(R)=S \otimes \rho^{T}(R)=S\left(\sigma^{+}\right)^{T} .
$$

Similarly $\sigma^{-\prime}=S\left(\sigma^{-}\right)^{T}$, and so the following matrices are obtained:

$$
\sigma^{+^{\prime}}=\left(\begin{array}{cc}
q^{-H / 2} & -q^{3 / 2}\left(q-q^{-1}\right) X^{+} \\
0 & q^{H / 2}
\end{array}\right), \quad \sigma^{{ }^{\prime}}=\left(\begin{array}{cc}
q^{H / 2} & 0 \\
q^{-3 / 2}\left(q-q^{-1}\right) X^{-} & q^{-H / 2}
\end{array}\right) .
$$

The $q$ factors can be removed by a renormalisation $X^{ \pm} \rightarrow \beta^{ \pm 1} X^{ \pm}$, for some $\beta \in C[[h]]$. The $R$-matrix for this representation is:

$$
R^{\rho^{\prime}}=\rho^{T} \otimes \rho^{T}\left(R_{U_{q} s(2)}\right)=\rho \otimes \rho(T \circ R),
$$

since $S_{\circ} \otimes S_{\circ}\left(R_{U_{q} s l(2)}\right)=R_{U_{q} s l(2)}$.

This is the structure given in [7], under an appropriate change in normalisation of $X^{ \pm}$.

\section{The Dual to the Quantum Double}

The dual to the quantum double is introduced in this section since there exists a basis of this Hopf algebra that is particularly suited to the quotient structure induced by the Borel subalgebras of $U_{q} g$. The dual to the quantum double is also important since it allows us to prove that the quantum double itself can be constructed from the dual algebra $U_{q} g^{\prime}$. This establishes that the constructions of $\sigma^{ \pm}$are consistent, i.e. that they are independent of the arbitrary isomorphism $\vartheta^{\prime}$. The remaining commutation relation between $\sigma^{+}$and $\sigma^{-}(20)$ is derived in this section.

Recall that the quantum double $D\left(U_{q} b_{+}\right)$has two Hopf subalgebras $U_{q} b_{+}, U_{q} b_{-}^{\prime}$ and a quotient mapping to $U_{q} g$. If we denote the dual to the quantum double by $D^{\prime}\left(U_{q} b_{+}\right)$, then Theorem 1 implies that $D^{\prime}\left(U_{q} b_{+}\right)$has the following structure:

Quotient Structure:

$$
\begin{aligned}
& D^{\prime}\left(U_{q} b_{+}\right) \stackrel{\pi^{+}}{\longrightarrow} \frac{D^{\prime}\left(U_{q} b_{+}\right)}{U_{q} b_{+}^{\perp}} \cong U_{q} b_{+}^{\prime} \cong U_{q} b_{+}, \\
& D^{\prime}\left(U_{q} b_{+}\right) \stackrel{\pi^{-}}{\longrightarrow} \frac{D^{\prime}\left(U_{q} b_{+}\right)}{U_{q} b_{-}^{\perp}} \cong U_{q} b_{-} .
\end{aligned}
$$

Hopf Subalgebra Structure:

$$
U_{q} g^{\prime} \subset D^{\prime}\left(U_{q} b_{+}\right) \text {. }
$$

Here the quotient maps $\pi^{ \pm}$are understood as those corresponding to the ideals of the quantum double. These quotient homomorphisms will not be distinguished from those corresponding to $U_{q} g^{\prime}$. The quotient structure of $U_{q} g^{\prime},(10)$ is induced on restriction to the Hopf subalgebra $U_{q} g^{\prime}$ of $D^{\prime}\left(U_{q} b_{+}\right)$. 
Note that due to the original isomorphism $U_{q} b_{+} \otimes U_{q} b_{-}^{\prime} \cong D\left(U_{q} b_{+}\right)$(as a $C[[h]]$-module), Sect. 2 , the projection $\pi^{-}$takes $D^{\prime}\left(U_{q} b_{+}\right)$to $U_{q} b_{-}$.

Define the following dual bases:

where

$$
\begin{array}{ll}
\left\{\zeta_{s}\right\} \in U_{q} b_{+}, & \left\{\eta^{s}\right\} \in U_{q} b_{+}^{\prime}, \quad\left(\eta^{s}, \zeta_{t}\right)=\delta_{t}^{s}, \\
\left\{\eta_{s}\right\} \in U_{q} b_{-}, & \left\{\zeta^{s}\right\} \in U_{q} b_{-}^{\prime}, \quad\left(\zeta^{s}, \eta_{t}\right)=\delta_{t}^{s},
\end{array}
$$

$$
\eta_{s}=\vartheta^{-1}\left(\zeta_{s}\right), \quad \zeta^{s}=\vartheta^{\prime}\left(\eta^{s}\right)
$$

The quantum double $D\left(U_{q} b_{+}\right)$has a basis $\left\{\zeta_{t} \zeta^{s}\right\}^{8}$, since it is isomorphic (as a $C[[h]]$-module) to the tensor product $U_{q} b_{+} \otimes U_{q} b_{-}^{\prime} . D^{\prime}\left(U_{q} b_{+}\right)$is isomorphic (as a $C[[h]]$-module) to the tensor product $U_{q} b_{+}^{\prime} \otimes U_{q} b_{-} \subset\left(U_{q} b_{+} \otimes U_{q} b_{-}^{\prime}\right)^{\prime}$; hence a suitable basis for this algebra is $\left\{\eta^{t} \eta_{s}\right\}$, where the elements $\eta^{t}, \eta_{s}$. have their definitions extended to:

$$
\eta^{u}\left(\zeta_{t} \zeta^{s}\right)=\delta_{t}^{u} \delta_{0}^{s}, \quad \eta_{p}\left(\zeta_{t} \zeta^{s}\right)=\delta_{t}^{0} \delta_{p}^{s}
$$

We also note that $\eta^{0}=\eta_{0}=1$ is the identity map. This definition is dual to the process of embedding $U_{q} b_{+}, U_{q} b_{-}^{\prime}$ in the quantum double $D\left(U_{q} b_{+}\right)$as Hopf subalgebras.

The chosen bases are dual, that is:

$$
\eta^{u} \eta_{v}\left(\zeta_{t} \zeta^{s}\right)=\delta_{t}^{u} \delta_{v}^{s}
$$

In passing we note that the following Hopf structure holds in $D^{\prime}\left(U_{q} b_{+}\right)$:

1. $\eta_{s}, \eta^{t}$ have an identical algebra structure to the analogues in $U_{q} b_{-}, U_{q} b_{+}^{\prime}$,

2. $\eta_{s}, \eta^{t}$ commute,

3. the coalgebra structure of $\eta_{s}, \eta^{t}$ is the same as that in $U_{q} b_{-}, U_{q} b_{+}^{\prime}$ only on projection by the appropriate ideal.

Observe that the complications in the Hopf structure are now in the coalgebra, while those in the quantum double are in the algebra, (35) and [6].

The Hopf ideals can now be explicitly written down:

$$
\begin{aligned}
& U_{q} b_{+}^{\perp}=\left\{\eta^{u} \eta_{v} \in D^{\prime}\left(U_{q} b_{+}\right): u \geqq 0, v>0\right\}, \\
& U_{q} b_{-}^{\perp}=\left\{\eta^{u} \eta_{v} \in D^{\prime}\left(U_{q} b_{+}\right): u>0, v \geqq 0\right\} .
\end{aligned}
$$

In particular: $\eta^{s} \notin U_{q} b_{+}^{\perp}, \eta_{s} \notin U_{q} b_{-}^{\perp}$. Thus the quotient structure is:

$$
\pi^{+}\left(\eta^{s} \eta_{t}\right)=\delta_{0 t} \eta^{s}, \quad \pi^{-}\left(\eta^{s} \eta_{t}\right)=\delta^{0 s} \eta_{t}
$$

The following diagram is commutative:

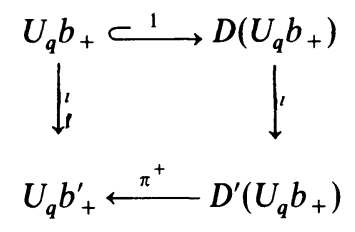

${ }^{8}$ This is shorthand for the more clear expression $\left\{\zeta_{t} \otimes \zeta^{s}\right\}$ 
The map $\imath$ is a duality map, taking an element to its dual. For example, for the Borel Hopf algebra $U_{q} b_{+}, l: \zeta_{s} \rightarrow \eta^{s}$. Hence we obtain: $l^{\circ} \pi^{+}\left(\eta^{s}\right)=\zeta_{s}$. But we also have: $\vartheta^{\circ} \pi^{-}\left(\eta_{s}\right)=\zeta_{s}$ from (30) and (31). This implies that if we define an algebra anti-isomorphism on the dual $D^{\prime}\left(U_{q} b_{+}\right)$by:

$$
\tilde{\imath}: \eta_{s}, \eta^{t} \rightarrow \eta^{s}, \eta_{t}
$$

then the following diagram is commutative:

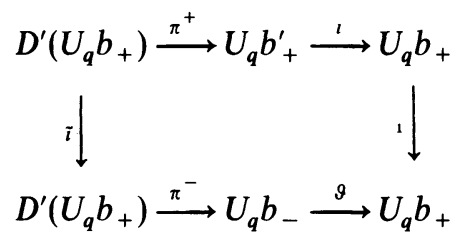

Fig. 3

Hence the following is equivalent to the quantum double construction:

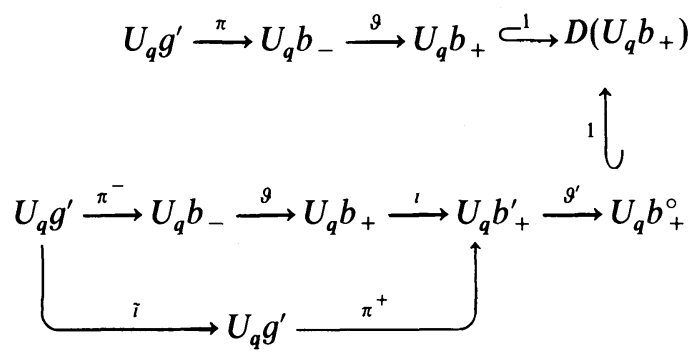

Fig. 4

The two mappings produce the dual bases $\left\{\vartheta^{\circ} \pi^{-}\left(\eta_{t}\right)\right\},\left\{\vartheta^{\prime} \circ \pi^{+} \circ \tilde{l}\left(\eta_{t}\right)\right\}$ for the Hopf subalgebras $U_{q} b_{+}$and $U_{q} b_{+}^{\circ}$ of $D\left(U_{q} b_{+}\right)$. Since the Hopf homomorphism $\pi: D\left(U_{q} b_{+}\right) \rightarrow U_{q} g$ is unique once $\left.\pi\right|_{U_{q} b_{+}}$is given, $[4,6]$ we deduce that the combinations $\pi_{g^{\circ}} \vartheta^{\circ} \pi^{-}$and $\pi_{g^{\circ}} \vartheta^{\prime} \circ \pi^{+}$are $\vartheta$ independent, (up to the arbitrary isomorphism $\left.\left.\pi\right|_{U_{q} b_{+}}\right)$. The notation $\pi_{\vartheta}$ stresses the $\vartheta$ dependence of $\pi$. Recalling that $\sigma^{+}=\pi_{\vartheta^{\circ}} \vartheta^{\prime} \circ \pi^{+}$and $\sigma^{-}=S \circ \pi_{g^{\circ}} \vartheta^{\circ} \pi^{-}$implies that $\sigma^{ \pm}$are independent of $\vartheta$, a conclusion also indicated in Eqs. (15), (16). This $\vartheta$-independence (up to the arbitrary isomorphism $\left.\pi\right|_{U_{q^{b}}+}$ ) is necessary for the construction to be reasonable, since $\vartheta$ is not intrinsic to the quantised algebra $U_{q} g$.

The algebra structure of the $\sigma^{ \pm}$generators will now be derived from $D^{\prime}\left(U_{q} b_{+}\right)$. The representation $\rho$ is extended to a representation of the quantum double $\rho: D\left(U_{q} b_{+}\right) \rightarrow \operatorname{End}(V, K)$ such that $\rho$ quotients through $U_{q} g$. The starting point is the definition of multiplication in the dual:

$$
\begin{aligned}
\left(\sigma_{i j}^{ \pm} \sigma_{k l}^{ \pm}, \eta\right) & =\left(\sigma_{i j}^{ \pm} \otimes \sigma_{k l}^{ \pm}, \Delta \eta\right)=\rho_{i j} \otimes \rho_{k l} \vartheta^{\prime} \pi^{+} \otimes \vartheta^{\prime} \pi^{+}(\Delta \eta), \quad(+) \\
& =\rho_{i j} \otimes \rho_{k l} \circ \vartheta \pi^{-} \otimes S \vartheta \pi^{-}(\Delta \eta), \quad(-) \quad \forall \eta \in D^{\prime}\left(U_{q} b_{+}\right) .
\end{aligned}
$$


Hence the commutation structure of $\sigma^{ \pm}$can be obtained by relating $\Delta \eta$ and $T \circ \Delta \eta$ for a general element $\eta \in D^{\prime}\left(U_{q} b_{+}\right)$. Since the action of the projectors $\pi^{ \pm}$is known on the base elements (31), we shall expand $\Delta \eta$ as:

$$
\Delta \eta=\left(\Delta \eta, \zeta_{q} \zeta^{p} \otimes \zeta_{t} \zeta^{s}\right) \eta^{q} \eta_{p} \otimes \eta^{t} \eta_{s} .
$$

By acting on the objects in (33) with the two homomorphisms $g^{\prime} \circ \pi^{+}, S \circ \vartheta^{\circ} \pi^{-}$, mapping $D^{\prime}\left(U_{q} b_{+}\right) \rightarrow D\left(U_{q} b_{+}\right)$, we obtain:

$$
\begin{aligned}
\vartheta^{\prime} \circ \pi^{+} \otimes \vartheta^{\prime} \circ \pi^{+}(\Delta \eta) & =\eta\left(\zeta_{q} \zeta_{t}\right) \zeta^{q} \otimes \zeta^{t}=\eta\left(\zeta_{v}\right) m_{q t}^{v} \zeta^{q} \otimes \zeta^{t}, \\
\vartheta^{\circ} \pi^{-} \otimes \vartheta^{\circ} \pi^{-}(\Delta \eta) & =\eta\left(\zeta^{p} \zeta^{s}\right) \zeta_{p} \otimes \zeta_{s}=\eta\left(\zeta^{u}\right) \mu_{u}^{p s} \zeta_{p} \otimes \zeta_{s}, \\
\vartheta^{\prime} \circ \pi^{+} \otimes S \circ \vartheta \circ \pi^{-}(\Delta \eta) & =\eta\left(\zeta_{q} \zeta^{s}\right) \zeta^{q} \otimes S\left(\zeta_{s}\right),
\end{aligned}
$$

because from (30), (31): $\vartheta^{\prime} \circ \pi^{+}\left(\eta^{s}\right)=\zeta^{s} \in U_{q} b_{-}^{\prime}, \vartheta \circ \pi^{-}\left(\eta_{s}\right)=\zeta_{s} \in U_{q} b_{+}$. The matrices $m, \mu$ are the matrices of multiplication and comultiplication for $U_{q} b_{+}$. Since $\Delta\left(\zeta^{v}\right)=m_{t q}^{v} \zeta^{q} \otimes \zeta^{t}, \Delta\left(\zeta_{v}\right)=\mu_{v}^{p s} \zeta_{p} \otimes \zeta_{s}$ (from duality), the first two relations can be manipulated into a coalgebra form:

$$
\eta\left(\zeta_{v}\right) T \circ \Delta\left(\zeta^{v}\right), \quad \eta\left(\zeta^{u}\right) \Delta\left(\zeta_{u}\right)
$$

Returning to (32), the universal $R$-matrix can now be used to obtain the results in (19). For the mixed algebra relation, (20) it is necessary to consider the commutation structure of the quantum double, since we are required to relate the elements $\zeta_{q} \zeta^{s}, \zeta^{u} \zeta_{v}$. The commutation relations of the quantum double, between elements of the Hopf subalgebras $U_{q} b_{+}$, and $U_{q} b_{+}^{\circ}$ can be expressed in terms of the matrices $m, \mu$ as [6]:

$$
m_{u p}^{r} \mu_{t}^{p v} \zeta^{u} \zeta_{v}=\mu_{t}^{q w} m_{w s}^{r} \zeta_{q} \zeta^{s}
$$

Hence we multiply the expression $\eta\left(\zeta_{q} \zeta^{s}\right) \zeta^{q} \otimes S\left(\zeta_{s}\right)$ in the third relation of (34) by appropriate terms in order to generate the $m, \mu$ matrices required in (35). So:

$$
\begin{aligned}
\eta\left(\zeta_{q} \zeta^{s}\right) \zeta^{q} \otimes S\left(\zeta_{s}\right) \cdot\left(\zeta^{w} \otimes S \zeta_{w}\right) & =\eta\left(\mu_{t}^{q w} m_{w s}^{r} \zeta_{q} \zeta^{s}\right) \zeta^{t} \otimes S\left(\zeta_{r}\right) \\
& =\eta\left(\zeta^{u} \zeta_{v}\right) \mu_{t}^{p v} \zeta^{t} \otimes m_{u p}^{r} S\left(\zeta_{r}\right) \\
& =\left(\zeta^{p} \otimes S \zeta_{p}\right) \cdot \vartheta^{\prime} \circ \pi^{+} \otimes S \circ \vartheta \circ \pi^{-}(T \circ \Delta \eta) .
\end{aligned}
$$

Note that the occurrence of the antipode is necessary, such that the indices are in the correct order. On observing that $R^{-1}=S \otimes 1\left(\sum_{s} \zeta_{s} \otimes \zeta^{s}\right)$ (12), we return to
(32) to obtain:

$$
\left(\sigma_{i j}^{+} \sigma_{k l}^{-}, \eta\right)=\left(\rho_{i j} \otimes \rho_{k l}, T \circ R^{-1}\left(\vartheta^{\prime} \circ \pi^{+} \otimes S \circ \vartheta \circ \pi^{-}(T \circ \Delta \eta)\right) T \circ R\right) .
$$

Thus on rearranging as in Sect. 1, using the fact that $\rho$ is a representation and that $\eta$ is arbitrary, the structure:

$$
R_{21} \sigma_{1}^{+} \sigma_{2}^{-}=\sigma_{2}^{-} \sigma_{1}^{+} R_{21}, \quad R_{21}=\rho \otimes \rho(T \circ R),
$$

is obtained, as quoted in (20). 


\section{The Quantised Lie Algebras $A_{n}$}

The Universal $R$-matrices for the quantised Lie algebras ${ }^{9} A_{n}$ are derived in [2] and [14]. Hence the Hopf structure of $A_{n}$ can be expressed in the matrix form (6) by the construction of Sect. 3. In $[2,14]$ a system of generators for $A_{n}$ based on the full root system of $A_{n}$ is defined, this being contrasted to that of $[6,10]$ which only use the simple roots. This system of generators occurs as the matrix elements of $\sigma^{ \pm}$, and the relations (6) allow all the commutation relations to be systematically derived. The fact that all the commutation relations are of the form of the adjoint structure, (36), as defined in [2], shows that this adjoint structure is sufficient to describe the algebra structure of $A_{n}$, and justifies the original definition.

The definitions of [2] will be summarised here for clarity: Let $\boldsymbol{\Phi}^{+}$denote the positive roots.

The positive roots are ordered by the length of the minimal word in the Weyl group needed to generate it from the end root of the Dynkin diagram; $\alpha_{1}$. The length of the word for the root $\alpha=\sum_{s \in[i, j]} \alpha_{s} \in \Phi^{+}, j \geqq i$, is $\mu(\alpha)=(j+i)-2$. So $\alpha<\beta$
if $\mu(\alpha)<\mu(\beta)$.

The generators in each Borel subalgebra are ordered by the corresponding roots: $P_{\alpha}<O_{\beta}$ iff $\alpha<\beta, P_{\alpha}, O_{\beta}$ two arbitrary generators corresponding to the positive roots $\alpha, \beta$.

Define the ordered products: $\prod^{<}, \prod^{>}$, where the $<,>$denote an ascending and descending order of generators respectively, when read from left to right.

The adjoint map is defined by:

$$
\operatorname{ad}_{q} P_{\alpha} \cdot O_{\beta}=P_{\alpha} O_{\beta}-q^{(\alpha, \beta)} O_{\beta} P_{\alpha},
$$

for $\alpha<\beta$, with the anti-symmetry condition $\operatorname{ad}_{q} P_{\alpha} \cdot O_{\beta}=-\operatorname{ad}_{q} O_{\beta} \cdot P_{\alpha}$. The generators $e_{i}, f_{i}$ will be employed, the index $i$ corresponding to the simple roots of $g$. These are related to the generators used in the introduction and most of the literature by [2]:

$$
e_{i}=q^{H_{i} / 2} X_{i}^{+}, f_{i}=q^{-H_{i} / 2} X_{i}^{-} .
$$

The following generators are then defined (for each positive root $\alpha$ ) by:

$$
e_{\alpha}=\prod_{s \in[i, j-1]}^{<}\left(\operatorname{ad}_{q} e_{s}\right) \cdot e_{j}, f_{\alpha}=\prod_{s \in[i+1 j]}^{>}\left(\operatorname{ad}_{q} f_{s}\right) \cdot f_{i}
$$

The generators $e_{\alpha}, f_{\alpha}$ corresponding to the root $\alpha=\sum_{s=i}^{j} \alpha_{s}$ (for $i \leqq j, \alpha_{s}$ simple) are denoted by $e_{i j}, f_{j i}$ respectively. The order of the indices is suggestive of the definitions (37). Note that in this notation $e_{i i}=e_{i}, f_{i i}=f_{i}$. We shall also employ the definition $H_{i j}=\sum_{s=i}^{j} H_{s}$.

\footnotetext{
9 With an abuse of notation, the symbol $A_{n}$ will denote the quantised object corresponding to this Lie algebra, i.e. $U_{q} s l(n+1)$
} 
The universal $R$-matrix for $A_{n}$ takes the form [2]:

with:

$$
R_{A_{n}}=\exp \left(\frac{h}{2} \sum_{i j} a_{i j}^{-1} H_{i} \otimes H_{j}\right) \prod_{\alpha \in \Phi^{+}}^{<} E_{q^{-2}}\left(\lambda e_{\alpha} \otimes f_{\alpha}\right)
$$

$$
E_{q}(x)=\sum_{r=0}^{\infty} \frac{x^{r}}{[r ; q] !}, \quad[u ; q] !=\prod_{i=1}^{u}[i ; q], \quad[i ; q]=\frac{\left(1-q^{i}\right)}{(1-q)}
$$

Here $a_{i j}$ is the Cartan matrix and $\lambda=1-q^{-2} . E_{q}$ is a $q$-analogue exponential. The universal $R$-matrix can also be expressed with the generators in a reversed order [2]. This form will be important for calculating the matrix $\sigma^{-}(50)$ :

$$
R_{A_{n}}=\prod_{\alpha \in \Phi^{+}}^{>} E_{q^{-2}}\left(\lambda S\left(e_{\alpha}\right) \otimes S\left(f_{\alpha}\right)\right) \exp \left(\frac{h}{2} \sum_{i j} a_{i j}^{-1} H_{i} \otimes H_{j}\right) .
$$

This is proved from (38) by recalling that the antipode $S$ is an algebra anti-homomorphism and using the relation $S \otimes S(R)=R$. The (skew) antipode does not preserve the adjoint structure (36); hence it is necessary to define an alternative definition of the adjoint action. Define [2]:

$$
\operatorname{ad}_{q}^{\prime} P_{\alpha} \cdot O_{\beta}=P_{\alpha} O_{\beta}-q^{-(\alpha, \beta)} O_{\beta} P_{\alpha}, \quad \alpha<\beta .
$$

This differs by a $q \rightarrow q^{-1}$ transformation from the previous adjoint definition (36). The following system of generators is an alternative set to (37):

$$
e_{\alpha}^{\prime}=\prod_{s \in[i, j-1]}^{<}\left(\operatorname{ad}_{q}^{\prime} e_{s}\right) \cdot e_{j}, f_{\alpha}^{\prime}=\prod_{s \in[i+1, j]}^{>}\left(\operatorname{ad}_{q}^{\prime} f_{s}\right) \cdot f_{i} \text {. }
$$

The following can be proved by induction:

$$
S\left(e_{\alpha}\right)=-q^{2} q^{-\boldsymbol{H}_{\alpha}} e_{\alpha}^{\prime}, \quad S\left(f_{\alpha}\right)=-q^{-2 m} q^{\boldsymbol{H}_{\alpha}} f_{\alpha}^{\prime},
$$

for $\alpha \in \boldsymbol{\Phi}^{+}$, the sum of $m+1$ simple roots.

The fundamental representation of $U_{q} s l(n+1)$ will be used [6]:

$$
\rho\left(H_{i}\right)=E_{i, i}-E_{i+1, i+1}, \quad \rho\left(e_{i}\right)=q^{1 / 2} E_{i, i+1}, \quad \rho\left(f_{i}\right)=q^{1 / 2} E_{i+1, i},
$$

where $E_{i j}$ is the matrix with value one at position $i, j$ and zero elsewhere. It is necessary to evaluate the representations of $e_{\alpha}, f_{\alpha}$. Noting that $E_{s t} \cdot E_{u v}=\delta_{t u} E_{s v}$, these can be calculated to be:

$$
\begin{aligned}
& \rho\left(e_{i j}\right)=\rho\left(e_{i} e_{i+1} \ldots e_{j}\right)=q^{1 / 2(j-i+1)} E_{i, j+1} \\
& \rho\left(f_{i j}\right)=\rho\left(q^{-(j-i)} f_{j} f_{j-1} \ldots f_{i}\right)=q^{1 / 2(i-j+1)} E_{j+1, i}
\end{aligned}
$$

The matrix $\sigma^{+} \in \operatorname{Mat}\left(n+1, U_{q} b_{+}\right)$is defined as (15): $\sigma_{i j}^{+}=\operatorname{Id} \otimes \rho_{i j}\left(R_{A_{n}}\right)$. Once this is evaluated the $R$-matrix in this representation can be obtained by $R^{\rho}=\rho\left(\sigma^{+}\right)$. The most difficult part of evaluating $\sigma^{+}$is the treatment of the coroot dependent prefactor of $R(38)$ :

$$
\mathrm{Id} \otimes \rho\left(\exp \frac{h}{2} \sum_{i j} a_{i j}^{-1} H_{i} \otimes H_{j}\right) .
$$

For this it is required to know the inverse of the Cartan matrix. The only property 
that we require is that:

$$
a_{i j}^{-1}-a_{i j-1}^{-1}=\left\{\begin{array}{ll}
1-\frac{i}{n+1} & j \leqq i \\
\frac{-i}{n+1} & j>i
\end{array} .\right.
$$

This is valid for all $i, j=1$ to $n+1$. (Take $a_{i 0}^{-1}=0$ and $\left.a_{i, n+1}^{-1}=0\right)$.

Using this equation we may proceed to calculate $\operatorname{Id} \otimes \rho\left(\exp \frac{h}{2} \sum_{i j} a_{i j}^{-1} H_{i} \otimes H_{j}\right)$ : $\mathrm{Id} \otimes \rho\left(\sum_{i j} a_{i j}^{-1} H_{i} \otimes H_{j}\right)=\sum_{i j=1}^{n} a_{i j}^{-1} H_{i}\left(E_{j j}-E_{j+1 j+1}\right)$

$$
=\sum_{i=1}^{n} \sum_{j=1}^{n+1}\left(a_{i j}^{-1}-a_{i j-1}^{-1}\right) H_{i} E_{j j}=\sum_{i=1}^{n} H_{i} \sum_{j=1}^{i} E_{j j}-\sum_{i=1}^{n} \frac{i H_{i}}{n+1} I .
$$

$I$ is the identity matrix. Define the matrices $I_{i}=\sum_{j=1}^{i} E_{j j}$. Hence we have:

$$
\begin{aligned}
\mathrm{Id} \otimes \rho\left(\exp \frac{h}{2} \sum_{i j} a_{i j}^{-1} H_{i} \otimes H_{j}\right) & =\exp \left(\frac{h}{2} \sum_{i=1}^{n} H_{i} I_{i}\right) \exp \left(-\frac{h}{2} \sum_{i=1}^{n} \frac{i H_{i}}{n+1} I\right) \\
& =\exp \left(-\frac{h}{2} \sum_{i=1}^{n} \frac{i H_{i}}{n+1}\right) \prod_{s=1}^{n} K_{s}\left(q^{H_{s}}\right) .
\end{aligned}
$$

The matrices $K_{s}$ have been defined as: $K_{s}(x)=I+(x-1) I_{s}$. They are diagonal with $x$ in the first $s$ positions, and 1 in those remaining.

Returning to the universal $R$-matrix, (38), we can now complete the calculation of the matrix $\sigma^{+}$by projecting in the second position the non-coroot part to the representation space $\operatorname{End}(V)$ :

$$
\begin{aligned}
\operatorname{Id} \otimes \rho\left(\prod_{\alpha \in \Phi^{+}}^{<} E_{q^{-2}}\left(\lambda e_{\alpha} \otimes f_{\alpha}\right)\right) & =\prod_{1 \leqq i \leqq j \leqq n}^{<}\left(I+\lambda q^{1 / 2(i-j+1)} e_{i j} E_{j+1, i}\right) \\
& =I+\sum_{1 \leqq i \leqq j \leqq n} \lambda q^{1 / 2(i-j+1)} e_{i j} E_{j+1, i}
\end{aligned}
$$

It is essential that the generators are in an ascending order such that no cross terms occur in expanding the product. Compare this to the $R$-matrix in the form (39), used in the calculation of $\sigma^{-}$, which would generate cross terms.

Collecting together the results (43) and (44), the matrix $\sigma^{+}$can be evaluated. It takes the form:

$$
\sigma^{+}=q^{-\sum_{i=1}^{n} \frac{I H_{i}}{n+1}} \prod_{s=1}^{n} K_{s}\left(q^{H_{s}}\right)\left(I+\sum_{1 \leqq i \leqq j \leqq n} \lambda q^{1 / 2(i-j+1)} e_{i j} E_{j+1, i}\right) .
$$

It is advantageous to define the following combinations of elements due to their 
occurrence in the matrix $\sigma^{+}$:

$$
\omega_{k}=q^{-\sum_{i=1}^{n} \frac{i H_{i}}{n+1}} q^{\sum_{s=k}^{n} H_{s}}, \quad k=1 \ldots n, \quad \text { and } \quad \omega_{n+1}=q^{-\sum_{i=1}^{n} \frac{i H_{i}}{n+1}} .
$$

This allows the coroot dependent part to be written as:

$$
q^{-\sum_{i=1}^{n} \frac{i H_{i}}{n+1}} \prod_{s=1}^{n} K_{s}\left(q^{H_{s}}\right)=\sum_{k=1}^{n+1} \omega_{k} E_{k k}
$$

The matrix $\sigma^{+}$now takes the form:

$$
\sigma^{+}=\sum_{k=1}^{n+1} \omega_{k} E_{k k}+\sum_{1 \leqq i \leqq j \leqq n} \lambda q^{1 / 2(i-j+1)} \omega_{j+1} e_{i j} E_{j+1, i}
$$

The elements $\omega_{k}$ have the following algebra:

$$
\begin{aligned}
& \omega_{k} e_{k t}=q e_{k t} \omega_{k}, \quad \omega_{k+1} e_{s k}=q^{-1} e_{s k} \omega_{k+1}, \\
& \omega_{k} e_{s t}=e_{s t} \omega_{k} \quad \text { if } \quad s \neq k, t+1 \neq k,
\end{aligned}
$$

and a similar algebra under the transformation: $e_{s t} \rightarrow f_{t s}, \omega_{k} \rightarrow \omega_{k}^{-1}$.

Now consider calculating the matrix $\sigma^{-}$. The starting point is the expression (39) for the universal $R$-matrix, since this avoids the production of cross terms when expanding the following product:

$$
\begin{aligned}
\rho & \otimes \operatorname{Id}\left(\prod_{\alpha \in \Phi^{+}}^{>} E_{q^{-2}}\left(-q^{2} \lambda q^{-H_{x}} e_{\alpha} \otimes S\left(f_{\alpha}\right)\right)\right) \\
& =\prod_{1 \leqq i \leqq j \leqq n}^{>}\left(I-\lambda q q^{1 / 2(j-i+1)} S\left(f_{j i}\right) E_{i, j+1}\right) \\
& =I-q \lambda \sum_{1 \leqq i \leqq j \leqq n} q^{1 / 2(j-i+1)} S\left(f_{j i}\right) E_{i, j+1} .
\end{aligned}
$$

The expression (16) for $\sigma^{-}$implies that the quantity of interest is $\rho \otimes \operatorname{Id}(R)$. Using the above formulae (49) and (46), this becomes:

$$
\begin{aligned}
\rho \otimes \operatorname{Id}(R) & =\left(I-q \lambda \sum_{1 \leqq i \leqq j \leqq n} q^{1 / 2(j-i+1)} S\left(f_{j i}\right) E_{i, j+1}\right) \sum_{k=1}^{n+1} \omega_{k} E_{k k} \\
& =\sum_{k=1}^{n+1} \omega_{k} E_{k k}-q \lambda \sum_{1 \leqq i \leqq j \leqq n} q^{1 / 2(j-i+1)} S\left(f_{j i}\right) \omega_{j+1} E_{i, j+1} .
\end{aligned}
$$

We now deduce by operating with the antipodal map that:

$$
\sigma^{-}=\sum_{k=1}^{n+1} \omega_{k}^{-1} E_{k k}-q \lambda \sum_{1 \leqq i \leqq j \leqq n} q^{1 / 2(j-i+1)} \omega_{j+1}^{-1} f_{j i} E_{i, j+1} .
$$

The only remaining calculation to complete the matrix formulation of $U_{q} s l(n+1)$ is to find the $R$-matrix in this representation. The easiest method is to use the 
expression (47) for $\sigma^{+}$and $R^{\rho}=\rho\left(\sigma^{+}\right)$. The various components are:

$\rho\left(\sum_{i=1}^{n} \frac{i H_{i}}{n+1}\right)=I-(n+1) E_{n+1, n+1}, \rho\left(q^{-\sum_{i=1}^{n} \frac{i H_{i}}{n+1}}\right)=q^{-1 /(n+1)}\left(I+(q-1) E_{n+1, n+1}\right)$,

and thus:

$$
\rho\left(\prod_{s=1}^{n} K_{s}\left(q^{H_{s}}\right)\right)=I \otimes I+(q-1) \sum_{k=1}^{n} E_{k k} \otimes E_{k k}+\left(q^{-1}-1\right) \sum_{k=1}^{n} E_{n+1, n+1} \otimes E_{k k} .
$$

Throughout this calculation the element $E_{n+1, n+1}$ has to be treated separately. However this asymmetry cancels to give the final result:

$$
R_{\rho \otimes \rho}=q^{-1 /(n+1)}\left(I \otimes I+(q-1) \sum_{i=1}^{n+1} E_{i i} \otimes E_{i i}+\left(q-q^{-1}\right) \sum_{i<j} E_{i j} \otimes E_{j i}\right) .
$$

This was quoted in [2] and also given in [6].

To summarise: the universal $R$-matrix for $U_{q} s l(n+1)(38)$ is used to construct two triangular matrices valued in $U_{q} s l(n+1)$, defined by (15), (16):

$$
\sigma_{i j}^{+}=I d \otimes \rho_{i j}\left(R_{A_{n}}\right), \quad \sigma_{i j}^{-}=\rho_{i j} \otimes \operatorname{Id}\left(R_{A_{n}}^{-1}\right) .
$$

These are found to have the form (47), (50):

$$
\begin{aligned}
& \sigma^{+}=\sum_{k=1}^{n+1} \omega_{k} E_{k k}+\sum_{1 \leqq i \leqq j \leqq n} \lambda q^{1 / 2(i-j+1)} \omega_{j+1} e_{i j} E_{j+1, i}, \\
& \sigma^{-}=\sum_{k=1}^{n+1} \omega_{k}^{-1} E_{k k}-q \lambda \sum_{1 \leqq i \leqq j \leqq n} q^{1 / 2(j-i+1)} \omega_{j+1}^{-1} f_{j i} E_{i, j+1},
\end{aligned}
$$

where $\omega_{k}$ is defined in (45). These encode the Hopf structure of $U_{q} s l(n+1)$ in the matrix form (6). It is obvious from the above form for $\sigma^{ \pm}$that $\sigma^{+}$and $\sigma^{-}$generate isomorphic subalgebras: the Borel subalgebras. An isomorphism was given in Sect. 5; matrix transposition inducing the following transformation of generators:

$$
e_{i j} \leftrightarrow-q^{j+1-i} f_{j i} \quad \text { or } \quad e_{\alpha} \leftrightarrow-q^{m+1} f_{\alpha} .
$$

This can easily be verified to be an algebra isomorphism and anti-coalgebra isomorphism as stated in Sect. 5 .

The ordering of the generators required for the definition of the adjoint action and universal $R$-matrix used in [2] can be read off from the matrix $\sigma^{+}$. It is given by the orthogonal projection onto the main diagonal.

The results of expanding the matrices in (6), i.e. finding the commutation relations will now be stated. The proof is long and so omitted. Note that expressing the algebra in this matrix form allows all the commutation relations to be systematically derived. Only partial results were given in [2], only those that were required being calculated. All the commutation relations within a Borel subalgebra take the form of the adjoint structure; in particular there is no mixing between the two definitions of the adjoint (36), (40). This suggests that the adjoint structure (36) and generators (37) are intrinsic to the algebra structure of the quantised Lie 
algebra $A_{n}$. (Of course the alternative definition of adjoint (40) leads to a structure that is just as self consistent.)

In order to simplify the notation we shall treat the roots set theoretically with respect to the simple root decomposition using some base $S$, in addition to all previous interpretations and conventions. Hence the root $\alpha=\sum_{i=u}^{v} \alpha_{i}, v \geqq u$ will be considered as the set $\left\{\alpha_{i}\right\}_{i \in[u, v]}$.

Expanding $R_{21} \sigma_{1}^{ \pm} \sigma_{2}^{ \pm}=\sigma_{2}^{ \pm} \sigma_{1}^{ \pm} R_{21}$ gives:

For $\alpha+\beta \in \Phi^{+}, \alpha<\beta$,

$$
\begin{aligned}
& e_{\alpha+\beta}=\operatorname{ad}_{q} e_{\alpha} \cdot e_{\beta}=e_{\alpha} e_{\beta}-q^{-1} e_{\beta} e_{\alpha}, \\
& f_{\alpha+\beta}=-\operatorname{ad}_{q} f_{\alpha} \cdot f_{\beta}=-f_{\alpha} f_{\beta}+q^{-1} f_{\beta} f_{\alpha} .
\end{aligned}
$$

For $\alpha+\beta \notin \Phi^{+}$and $\alpha \cap \beta=\varnothing$ or $\alpha \subset \beta$ or $\beta \subset \alpha$,

$$
\begin{aligned}
\operatorname{ad}_{q} e_{\alpha} \cdot e_{\beta} & =e_{\alpha} e_{\beta}-e_{\beta} e_{\alpha}=0, \\
\operatorname{ad}_{q} f_{\alpha} \cdot f_{\beta} & =f_{\alpha} f_{\beta}-f_{\beta} f_{\alpha}=0, \quad \alpha<\beta .
\end{aligned}
$$

For $\alpha+\beta \notin \Phi^{+}$and $\alpha \cap \beta \neq \varnothing, \alpha=\bar{\alpha}+\gamma, \beta=\gamma+\bar{\beta}$,

$$
\begin{aligned}
\operatorname{ad}_{q} e_{\alpha} \cdot e_{\beta} & =e_{\alpha} e_{\beta}-e_{\beta} e_{\alpha}=\left(q-q^{-1}\right) e_{\gamma} e_{\bar{\alpha}+\beta}, \\
\operatorname{ad}_{q} f_{\alpha} \cdot f_{\beta} & =f_{\alpha} f_{\beta}-f_{\beta} f_{\alpha}=\left(q-q^{-1}\right) f_{\gamma} f_{\bar{\alpha}+\beta},
\end{aligned}
$$

with $\gamma \in \Phi^{+}, \alpha<\gamma<\beta$. Note that the pair $e_{\gamma}, e_{\bar{\alpha}+\beta}$ commute, and similarly for the pair of $f$ generators.

Expanding the relation $R_{21} \sigma_{1}^{+} \sigma_{2}^{-}=\sigma_{2}^{-} \sigma_{1}^{+} R_{21}$ produces:

For all roots $\alpha \in \Phi^{+}$,

$$
\left[e_{\alpha}, f_{\alpha}\right]=\frac{1}{\lambda}\left(q^{H_{\alpha}}-q^{-H_{\alpha}}\right) .
$$

For $\alpha \cap \beta=\varnothing$ or $\alpha \subset \beta, \beta-\alpha \notin \Phi^{+}$(i.e. $\beta$ encloses $\alpha$ ),

$$
\left[e_{\alpha}, f_{\beta}\right]=0,\left[e_{\beta}, f_{\alpha}\right]=0 \text {. }
$$

For $\alpha \cap \beta \neq \varnothing, \alpha=\beta+\gamma$,

$$
\begin{aligned}
& {\left[e_{\alpha}, f_{\beta}\right]=-q^{-\boldsymbol{H}_{\beta}} e_{\gamma}, \quad \beta<\gamma,} \\
& {\left[e_{\alpha}, f_{\beta}\right]=q q^{\boldsymbol{H}_{\beta}} e_{\gamma}, \quad \beta>\gamma .}
\end{aligned}
$$

For $\alpha \cap \beta \neq \varnothing, \beta=\alpha+\gamma$,

$$
\begin{array}{ll}
{\left[e_{\alpha}, f_{\beta}\right]=-q^{H_{\alpha}} f_{\gamma},} & \alpha<\gamma, \\
{\left[e_{\alpha}, f_{\beta}\right]=q q^{-H_{\alpha}} f_{\gamma},} & \alpha>\gamma .
\end{array}
$$

For $\alpha \cap \beta \neq \varnothing, \alpha=\bar{\alpha}+\gamma, \beta=\gamma+\bar{\beta}$,

$$
\begin{array}{ll}
{\left[e_{\alpha}, f_{\beta}\right]=-\left(q-q^{-1}\right) q^{H_{\gamma}} e_{\bar{\alpha}} f_{\bar{\beta}},} & \alpha<\beta, \\
{\left[e_{\alpha}, f_{\beta}\right]=\left(q-q^{-1}\right) q^{-H_{\gamma}} e_{\bar{\alpha}} f_{\bar{\beta}},} & \alpha<\beta .
\end{array}
$$

The algebra satisfied by the $\omega_{k}$ was given in (48). Alternatively the full Hopf 
structure with the generators $H_{i}$ can be used. This is reproduced for completeness:

$$
\begin{aligned}
& {\left[H_{\alpha}, e_{\beta}\right]=(\alpha, \beta) e_{\beta},} \\
& {\left[H_{\alpha}, f_{\beta}\right]=-(\alpha, \beta) f_{\beta} .}
\end{aligned}
$$
The coalgebra structure for $U_{q} g$ is given by the relations $\Delta \sigma_{i j}^{ \pm}=\sum_{k} \sigma_{i k}^{ \pm} \otimes \sigma_{k j}^{ \pm}$. It
can be verified that the following structure is obtained:

$$
\begin{aligned}
& \Delta e_{\alpha}=1 \otimes e_{\alpha}+e_{\alpha} \otimes q^{H_{\alpha}}+\left(q-q^{-1}\right) \sum_{\substack{\beta>\beta^{\prime} \\
\beta+\beta^{\prime}=\alpha \\
\beta, \beta^{\prime} \in \Phi^{+}}} e_{\beta} \otimes q^{H_{\beta}} e_{\beta^{\prime}}, \\
& \Delta f_{\alpha}=q^{-H_{\alpha}} \otimes f_{\alpha}+f_{\alpha} \otimes 1-\left(q-q^{-1}\right) \sum_{\substack{\beta>\beta^{\prime} \\
\beta+\beta^{\prime}=\alpha \\
\beta, \beta^{\prime} \in \Phi^{+}}} q^{-H_{\beta}} f_{\beta^{\prime}} \otimes f_{\beta}, \quad \forall \alpha \in \Phi^{+} .
\end{aligned}
$$

This is the structure originally given in [2].

\section{Conclusion}

In this paper the Hopf structure of the quantised algebra $U_{q} g$ is expressed in a matrix form (6) by defining two representations of the quantum group $U_{q} g^{\prime}$. In order to achieve this matrix formulation there are two requirements: we require a representation of the dual $U_{q} g^{\prime}$, this giving the coalgebra expression (8), and an universal $R$-matrix such that the algebra can be constructed (9). However the dual $U_{q} g^{\prime}$ is not quasi-triangular, Sect. 2 , and hence it is necessary to embed $U_{q} g^{\prime}$ into a quasi-triangular Hopf algebra, and use a representation of this algebra. The most obvious choice is the quantum double of $U_{q} g^{\prime}[4]$, denoted by $D\left(U_{q} g^{\prime}\right)$. The construction in Sect. 3 exploiting the quotient structure of the quantum group $U_{q} g^{\prime}(10)$, is interpretable in this fashion, that is, there exist two morphisms: $D\left(U_{q} g^{\prime}\right) \rightarrow U_{q} g$ which when restricted to $U_{q} g^{\prime}$ map onto the Borel subalgebras; $U_{q} b_{+}, U_{q} b_{-}$. These will be algebra homomorphisms, coalgebra anti-homomorphisms. Our construction gives an expression for the matrices of generators $L^{ \pm}$ defined in [7] in terms of the universal $R$-matrix of $U_{q} g$ and a representation of $U_{q} g,(15),(16)$. When using the fundamental representation of the quantised algebra $U_{q} s l(n)$, we deduce that the Borel subalgebras $U_{q} b_{ \pm}$are generated by $L^{ \pm}$, the quantum determinant condition arising naturally. The case for other quantised algebras is more complex; additional representations must be used since the fundamental representation no longer generates the function space of the associated Lie group. These cases are more easily treated by using the quantum double $D\left(U_{q} g\right)$, our construction deriving ultimately from the quasi-triangular structure of this Hopf algebra [4]. However the construction for general $U_{q} g$ will be very similar, matrices $L^{ \pm}$, being defined by (15), (16) for each irreducible representation required

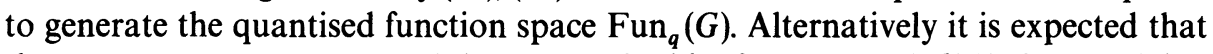
the quantum groups $\operatorname{Fun}_{q}(G)$ are attainable from $\operatorname{Fun}_{q}(G l(n))$ by applying functional constraints.

The matrix formulation of [7] naturally leads to a system of generators based on the whole root system, as in $[2,14]$, contrasting to that based only on the simple roots as used in $[6,10]$. The construction of the matrices $L^{ \pm}$for the general 
Lie algebras, [5] will produce a system of generators based on the whole root system, and should reproduce the adjoint structure presented in [15], an extension of that for $U_{q} s l(n),[2,14]$.

Acknowledgements. I am indebted to Alan Macfarlane for our many discussions on quantum groups, his help in preparing this manuscript and initiating this work by a question: why does the relation $R_{21} L_{1}^{(+)} L_{2}^{(-)}=L_{2}^{(-)} L_{1}^{(+)} R_{21}$ occur in the algebra of Faddeev et al and not the corresponding relation $R_{21} L_{1}^{(-)} L_{2}^{(+)}=L_{2}^{(+)} L_{1}^{(-)} R_{21}$ ? The answer proposed in this paper is that it is a consequence of the algebraic structure of the quantum double, that is, a consequence of the requirement that the quantum double is quasi-triangular.

On completion of this work, [8] and [11] came to the attention of the author. Paper [11] extends the analysis of [7], an universal $R$-matrix being defined via a homomorphism $A(R)$ to $U(R)$. Paper [8] considers the constructions of [7] from a universal aspect using quantum doubles. The relations (15) and (16) are obtained in both these works.

\section{References}

1. Abe, E.: Hopf Algebras: Cambridge: Cambridge University Press 1977

2. Burroughs, N. J.: The Universal $R$-matrix for $U_{q} s l(3)$ and Beyond! Commun. Math. Phys. 127, $109-128(1990)$

3. Burroughs, N. J.: The Quantum Group methods of quantising the Special Linear group $S l(2)$. DAMTP/R-89/16. Proceedings of the 1989 Banff Summer School on Physics, Geometry and Topology, H. C. Lee (ed.) (to appear)

4. Burroughs, N.: in preparation

5. Burroughs, $\mathrm{N}$.: in preparation

6. Drinfel'd, V. G.: Quantum Groups. ICM Berkeley 1986, A. M. Gleason (ed.). Providence RI: AMS, 1987 p. 798

7. Faddeev, L. Reshetikhin, N., Takhtajan, L.: Quantisation of Lie groups and Lie algebras. Leningrad preprint LOMI E-14-87

8. Faddeev, L., Reshetikhin, N., Takhtajan, L.: Quantum groups. in: Braid Group, Knot Theory and Statistical Mechanics. Yang, C. N., Ge, M. L. (eds.). Singapore: World Scientific 1989

9. Jimbo, M.: A $q$-Difference Analogue of $U(g)$ and the Yang-Baxter Equation. Lett. Math. Phys. 10, 63-69 (1985)

10. Jimbo, M.: A $q$-analogue of the $U_{q}(g l(N+1))$. Hecke Algebra, and the Yang-Baxter Equation. Lett. Math. Phys. 11, 247-252 (1986)

11. Majid, S.: Quasitriangular Hopf Algebras and Yang-Baxter Equations. Int. J. Mod. Phys. A5, 1-91 (1990)

12. Manin, Yu. I.: Some remarks on Koszul algebras and quantum groups. Ann. Inst. Fourier, Tome XXXVII, F. 4, 191-205 (1987)

13. Manin, Yu. I.: Quantum groups and non-commutative geometry. Preprint Montreal University, CRM-1561, 1988

14. Rosso, M.: An analogue of P.B.W. Theorem and the Universal $R$-matrix for $U_{h} s l(N+1)$. Commun. Math. Phys. 124, 307-318 (1989)

15. Takeuchi, M.: The $q$-bracket product and the PBW theorem for quantum enveloping algebras of classical types $A_{n}, B_{n}, C_{n}$ and $D_{n}$. Institute of Mathematics, University of Tsukuba preprint

16. Woronowicz, S. L.: Compact Matrix Pseudogroups. Commun. Math. Phys. 111, 613-665 (1987)

17. Woronowicz, S. L.: Pseudospaces, pseudogroups and Pontriagin duality, Proceedings of the International Conference on Mathematics and Physics Lausanne 1979. Lecture Notes in Physics vol. 116. Berlin, Heidelberg, New York: Springer 1980

Communicated by J. Fröhlich 
